\title{
The brain in flow: a systematic review on the neural basis of the flow state
}

Clara Alameda ${ }^{1,2}$, Daniel Sanabria ${ }^{1,2} \&$ Luis F. Ciria ${ }^{1,2 *}$

${ }^{1}$ Mind, Brain \& Behavior Research Center, University of Granada, Spain

${ }^{2}$ Department of Experimental Psychology, University of Granada, Spain

*Corresponding author: 1ciria@ugr.es 


\section{Abstract}

\section{Background:}

3

4

Flow state is a subjective experience that people report when task performance is experienced as automatic, intrinsically rewarding, optimal and effortless. While this intriguing phenomenon is the subject of a plethora of behavioural studies, only recently researchers have started to look at its neural correlates. Here, we aim to systematically and critically review the existing literature on the neural correlates of the flow state.

\section{Methods:}

Three electronic databases (Web of Science, Scopus and PsycINFO) were searched to acquire information on eligible articles in July, 2021, and updated in March, 2022. Studies that measured or manipulated flow state (through questionnaires or employing experimental paradigms) and recorded associated brain activity with electroencephalography (EEG), functional magnetic resonance (fMRI) or functional near-infrared spectroscopy (fNIRS) or manipulated brain activity with transcranial direct stimulation (tDCS) were selected. We used the Cochrane Collaboration Risk of Bias 2 ( RoB 2) tool to assess the methodological quality of eligible records.

\section{Results:}

In total, 25 studies were included, which involved 471 participants. In general, the studies that experimentally addressed flow state and its neural dynamics seem to converge on the key role of structures linked to attention, executive function and reward systems, giving to the anterior brain areas (e.g., the DLPC, MPFC, IFG) a crucial role in the experience of flow. However, the dynamics of these brain regions during flow state are inconsistent across studies.

\section{Discussion:}

In light of the results, we conclude that the current available evidence is sparse and inconclusive, which limits any theoretical debate. We also outline major limitations of this literature (the small number of studies, the high heterogeneity across them and their important methodological constraints) and highlight several aspects regarding experimental design and flow measurements that may provide useful avenues for future studies on this topic.

Keywords: consciousness, cognitive processing, phenomenology, EEG, fMRI, tDCS, fNIRS 


\section{Introduction}

Have you ever been so focused on a task that you stopped noticing what was going on around you? Were you exercising, playing an instrument or working and felt that it took less effort than usual, or that time flew by? If so, then you have experienced what Csikszentmihalyi (1975) called "flow state", a subjective experience in which the person is absorbed in the task and action seems to occur smoothly and automatically. The interest in this phenomenon has resulted in a wealth of literature investigating its subjective and behavioural manifestation (e.g., Csikszentmihalhi, 2020; Ottiger et al., 2021; Swann et al., 2012), albeit much less research has been conducted to pinpoint its neural signatures. Here, we systematically review the extant evidence on the neural correlates of flow state from a critical perspective, highlighting both the advances in the field and what we consider major limitations.

Investigating the flow state, also known as "being in the zone", has theoretical and practical relevance. At the theoretical level, flow state has been described as a particular state of consciousness, related to core cognitive processes such as vigilance and attention (Csikszentmihalyi et al., 2021). On a practical level, the state of flow has long raised the attention of researchers because of its potential role in linking feelings of enjoyment and subjective well-being, with optimal cognitive and physical performance (e.g., Flett, 2015; Khoshnoud et al., 2020). For instance, in a 10-year longitudinal study in which over 5000 executives were asked about their flow experiences at work, Cranston and Keller (2013) showed that people in flow state reported increasing their productivity by $500 \%$. An improvement in performance associated with the flow state has also been observed in other contexts, such as sports (e.g., Stavrou et al., 2007) and music (e.g., MacDonald et al., 2006).

When defining the flow state, the majority of studies based on subjective measures (e.g., questionnaires or verbal reports) agree on the following nine dimensions (e.g., Nakamura and Csikszentmihalyi, 2014): 1) clear goals, 2) high level of concentration on the task, 3) balance between the individual's skills and task difficulty, 4) immediate feedback about performance, 5) sense of control, 6) fusion of action and consciousness or automaticity, 7) autotelic property or intrinsically rewarding activity, 8) changed experience of time, and 9) decreased selfconsciousness and absence of worry. In addition to these classical experiential factors, certain facilitating conditions seem also necessary for the flow state to arise (e.g., Lambert and Csikszentmihalyi, 2020). In general, the state of flow is usually experienced by highly motivated experts (e.g., surgeons) with traits of autotelic personality, carrying out critical tasks 
(e.g., a surgery) in well-known task-related scenarios (e.g., operating room) during prolonged periods of time without interruption.

Even if anyone can achieve a state of flow when the conditions described above (e.g., balance between difficulty and skill) are met, there are individual differences in the ease/frequency with which people enter this state (Schmidt et al., 2014). Overall, current evidence suggests that differences in the tendency to experience flow may be determined by autotelic personality traits (Ross \& Keiser, 2014), genes associated with the neurotransmitter dopamine receptors (Gyurkovics et al., 2016), and social and educational factors (Heo et al., 2010). High self-esteem, low neuroticism, high extraversion, higher school support, higher employment status, higher availability of D2R in the striatum and CC homozygotes of the DRD2 C957T SNP gene are associated with more frequent experiences of flow (Butkovic et al., 2015; de Manzano et al., 2013; Gyurkovics et al., 2016; Heo et al., 2010; Mosing et al., 2012; Ullén et al., 2016). In fact, Mosing and collaborators (2012) estimated a heritability of $41 \%$ for general flow proneness. Paradoxically, even if all the above conditions are met in a person who is prone to experiencing flow, flow state does not necessarily arise. Eliciting and capturing the flow state therefore seem rather elusive, even more so in controlled laboratory conditions (e.g., with an individual inside the functional magnetic resonance imaging -fMRIscanner). Nevertheless, researchers have attempted to investigate the neural basis of flow.

In search of the neural correlates of flow state, several brain systems and networks have been pointed out as potential neural underpins of this experience (see van der Linden et al., 2021, for a brief summary): a) the reward system, a mesocorticolimbic circuit which includes amygdala, hippocampus, nucleus accumbens, and ventral diencephalon (e.g., hypothalamus), as well as cortical areas (e.g., dorsolateral prefrontal and cingulate cortices, the insula), critically involved in positive or negative reinforcement and motivation processes (Makris et al., 2008); b) attentional networks, such as the orienting network —related to the selection of targets towards which the attentional focus is directed, and that includes the superior parietal cortices, the temporal-parietal junction and the frontal eye fields (Posner \& Rothbart, 2007) and the alerting network - associated with the increases and maintenance of the attentional level, and that includes the locus coeruleus and right frontal and parietal cortices (Posner \& Rothbart, 2007)—; and c) the default mode network, a complex set of brain regions (i.e., anterolateral middle temporal cortex, posteriomedial cortex, angular gyrus, inferior frontal gyrus and medial prefrontal cortex) involved in both resting and mind-wandering states, as well 
as in higher-order cognitive processes (e.g., decisions based on internal rules; Smallwood et al., 2021). These neural systems seem to be involved in some of the conditions traditionally associated with the state of flow, such as its autotelic property, high levels of concentration and attention, or the influence of previous experiences. The fundamental question hence is whether the current evidence provides solid support for the involvement of any of these networks, or any other brain regions in the generation of the flow experience.

The findings of the studies searching for the neural basis of flow have been traditionally framed along with two main theoretical accounts: the Transient Hypofrontality Hypothesis (THH; Dietrich, 2004) and the Synchronization Theory of Flow (STF; Weber et al., 2009). According to the former, flow state requires the support of implicit and automatic systems, including the basal ganglia and cerebellum, as well as the inhibition of most cognitive functions linked to prefrontal areas (considered more explicit systems; Dietrich, 2004). On the other hand, the STF, based on Posner's (1987) tripartite attentional model, suggests that flow state arises from the synchronization of focused attention networks (alertness and visual orienting networks) together with the striatal reward networks, whose activation would allow the pleasurable component of flow state to rise. Very recently, in an attempt to reconcile these two seemingly opposite explanations of the flow state, Gold and Ciorciari (2021) developed a more comprehensive neural model of flow: the Internal Model of Flow. According to this framework, the underlying neural mechanism of flow state relies on internal models formed in the cerebellum during the acquisition of cognitive or motor skills. When an individual is experiencing flow, the orders to execute the actions would come, in first place, from frontal areas (e.g., the premotor cortex, the pre-supplementary motor cortex or the anterior cingulate cortex). These instructions, instead of being executed by the prefrontal cortex (if the task is cognitive) or the motor cortex (if it implies physical activity), would be carried out by the cerebellum when in flow. This cerebellar control would be responsible for the experience of intuitive and effortless behaviour. Thus, a secondary question addressed by the present paper would be to explore whether current research on the neural basis of flow provides support for any of these theoretical models.

The aim of the present systematic review is to synthesise and organise the current evidence on the neural correlates of flow state. To this end, we searched for studies using neuroimaging techniques such as electroencephalography (EEG), fMRI or functional nearinfrared spectroscopy (fNIRS) to measure brain activity, and transcranial direct current 
stimulation (tDCS) to modulate neural activity while inducing or assessing the experience of flow. The results are critically summarized considering the latest theoretical accounts of the flow state, the shortcomings of the investigation up to now, and what, in our opinion, are key methodological issues to address by future research.

\section{Methods}

This systematic literature review followed the Preferred Reporting for Items for Systematic Reviews and Meta-Analyses (PRISMA) guidelines (Moher et al., 2015).

\subsection{Literature search}

In July 2021, and again in March 2022 (to update the search after the first round of reviews), the electronic databases Web of Science (main collection), Scopus and PsycINFO were searched for relevant studies by a string that combined the following terms: ("flow stat*" OR "flow experienc*" OR "experienc* of flow" OR "stat* of flow" OR "flow engagement" OR "flow-like stat*" OR "psychological flow" OR "theory of flow" OR "cognitive flow" OR "being in the zone" OR "flow research") AND (EEG OR fMRI OR tDCS OR TMS OR FNIRS) NOT ("optic flow" OR "current flow" OR "airflow"). The literature search was narrowed to the title, abstract or keywords of original published studies written in English language. Additional titles were identified by a manual search of reference sections of topic-relevant papers and citations to them by other papers.

\subsection{Eligibility criteria}

Studies were considered eligible for inclusion if they met the following criteria: (a) studies that measured or manipulated flow state (through questionnaires or employing experimental paradigms) and recorded associated brain activity; (b) peer-reviewed papers or preprints with a DOI. Exclusion criteria were: (a) exploratory studies that did not measure brain activity during the flow state; (b) systematic or narrative reviews on the psychophysiology of the flow state; (c) studies with clinical samples. We did not establish any restrictions on publication date. 
157

158

159

160

161

162

163

164

165

166

167

168

169

170

171

172

173

174

\subsection{Study selection}

After conducting electronic database searches, we pooled results and removed duplicate articles by using Mendeley software. Next, articles with irrelevant titles and abstracts were excluded. Then, the excluded articles were examined by the last author (LC) to double-check their relevance for this review (none of the excluded articles was included again in the pool of relevant studies). The first (CA) and the last author (LC) assessed and judged independently the full texts of potentially relevant studies with respect to eligibility (see flowchart in Fig. 1 for details; see Excluded studies, in Supplementary material for details, i.e., references and reasons for exclusion). In case of disagreement, as was the case for 4 articles, the second author (DS) was consulted to reach a consensus.

Relevant information from all the included studies was retrieved by the first author using a data extraction table form developed a priori (see Table 1). Extracted data include: (i) characteristics of the study sample; (ii) experimental paradigm used; (iii) flow measures; (iv) brain activity technique; (v) main findings.

\subsection{Risk of bias}

A slightly modified version of the RoB 2 tool (revised Cochrane risk of bias tool for randomised trials; Sterne et al., 2019) was used to estimate the risk of bias in the 25 selected studies (see Risk of bias, in Supplementary material). 


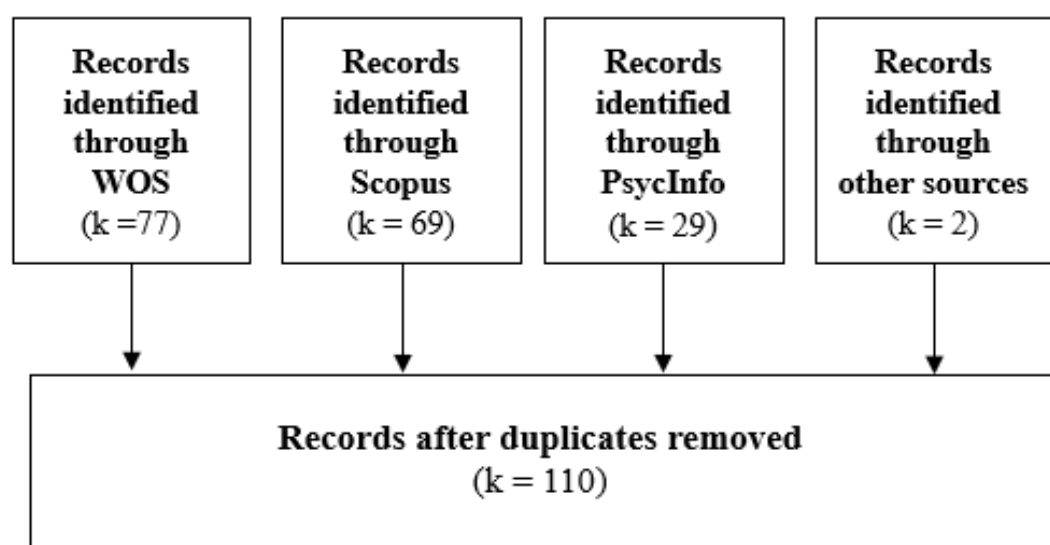

Studies excluded because titles or

abstract were not relevant to neural basis of flow state $(k=61)$

Full-text articles assessed for eligibility $(\mathrm{k}=49)$

Figure 1. PRISMA flow chart for study inclusion

3. Results

Our initial search, which was carried out for the first time in July 2021, yielded 122 eligible records. The search in March 2022 resulted in the inclusion of 55 further studies, resulting in a total of 177 eligible records (see Fig. 1). Then, we eliminated 67 duplicate records. 
From the remaining 110 studies, we excluded 61 studies non-related with the neural correlates of flow state or reviews about this topic, and 24 because they did not fulfil eligibility criteria (see figure 1, for details). Finally, 25 studies were included in this systematic review.

\subsection{Research on cerebral blood flow: fMRI and perfusion MRI}

Within the studies included in this systematic review, there are 7 studies that used neuroimaging techniques (fMRI and perfusion) to measure brain activity while (trying to) inducing flow state through different experimental protocols (Ju and Wallraven, 2019; Klasen et al., 2012; Huskey et al., 2018a; Huskey et al., 2018b; Ulrich et al., 2014; Ulrich et al., 2016a, 2016b).

\section{Difficulty-based studies}

The best example of this approach is the landmark study conducted by Ulrich and collaborators (2014) employing a mental arithmetic task to induce flow in 27 healthy male adults while recording their brain activity with fMRI. Participants were instructed to perform sums varying in difficulty. The difficulty was manipulated by adding or removing one -or two- digit numbers in the incoming operation to create 3 different experimental conditions where the difficulty of the sums could be a) excessively easy compared to the participant's skills level: the "boredom condition" (as it was not challenging enough and, therefore, flow was unlikely to emerge); b) excessively difficult compared with the participant's skills: the "overload condition"; c) dynamically adjusted to participant's ongoing performance: the "flow condition". Three blocks of each condition (lasting 184 seconds each) were performed in a random order. After each block, participants completed 9 Likert-scaled flow-related items to assess their subjective experience (desire to solve calculations again, feeling involved, thrilled, bored, or focused, having the necessary skills, ability-difficulty balance, number of taskrelevant thoughts, and subjective time experience). The results revealed increased cerebral blood flow in the left inferior frontal gyrus (IFG) and putamen, as well as in posterior cortical regions during what they called flow condition, relative to the boredom and overload conditions. In addition, they report reduced activity in the medial prefrontal cortex (MPFC) during flow state compared with overload and boredom conditions. Interestingly, they found that the neural activity in the IFG and the amygdala correlated with the subjective experience of flow. The authors suggest that the relative increase of neural activity found in the IFG which 
correlated with the subjective experience of flow might be associated with a high sense of control during the task, one of the main features of the flow state. However, increased activity

214 in the IFG has been also associated with mental arithmetic solving (e.g., Arsalidou and Taylor,

215 2011; Baldo and Dronkers, 2007; Zago et al., 2008), especially when task difficulty is high

216 (e.g., Gruber et al., 2001; Kong et al., 2005). On the other hand, the authors point to arousal as

217 a potential mechanism responsible for the reduced activity found in the amygdala, which may

218 reflect a decreased arousal level during flow state (e.g., Lewis et al., 2007; McGaugh, 2004),

219 although it would not explain why it was different from the boredom condition which should also be associated with even lower arousal.

221

222

223

224

225

226

227

228

229

230

231

232

233

234

235

236

237

238

239

Although these results were partially replicated in a subsequent experiment by the same authors (Ulrich et al., 2016a, 2016b), several methodological issues compromise the validity of these findings. Firstly, this paradigm is based exclusively on one of the 9 flow dimensions: skills-difficulty balance. That is, it is inferred that the flow state will naturally arise in a situation in which the difficulty of the task (i.e., performing sums) and the mathematical skills of the subject are balanced. However, the complex nature of flow state implies that it does not necessarily emerge in situations where skills and task difficulty are balanced (i.e., skillsdifficulty match is a necessary condition but not sufficient). Other key factors such as autotelic ownership or intrinsically rewarding activity seem necessary for the flow state to emerge (Nakamura \& Csikszentmihalyi, 2009). Secondly, performing sums is perhaps not as intrinsically rewarding as other tasks (i.e., playing video games). Thirdly, the flow experience appears to emerge while engaged in tasks lasting longer than 3-min (Csikszentmihalhi, 2020). Thus, performing a mathematical task —often associated with negative emotions in students (Lewis, 2013) - for blocks of such short duration (184 seconds) does not seem the ideal scenario for inducing flow state. Fourthly, the experimental sessions were carried on in an MRI scanner, an unusual, claustrophobic and noisy situation for most people which would definitely hinder the likelihood of experiencing flow. Lastly, in addition to the methodological limitations more closely related to the induction of the flow state, the results of these studies could have been also undermined by a notable risk of bias (e.g., significances were not corrected for multiple comparisons; see Risk of bias, in Supplementary material).

\section{Dual-task studies}

Huskey and collaborators (2018b, 2018a) followed a different approach to explore the neural correlates of flow state using a dual-task paradigm. This approach would eventually lead 
to two separate articles with different levels of analysis of brain activity (Huskey, Craighead, et al., 2018; Huskey, Wilcox, et al., 2018). In that experiment, 18 healthy young participants were required to play an experimental video game with three difficulty conditions (i.e., low,

247 balanced and high), each lasting for 120s, while performing a secondary visual reaction time

248 (RT) task. In line with their hypotheses, participants reported higher intrinsic reward sensitivity 249 (using the autotelic personality subscale of the Activity Experience Scale; Jackson and Eklund, 250 2002) in the balanced-difficulty condition (considered as flow condition based on the same idea

251

252

253

254

255

256

257

258

259

260

261

262

263

264

265

266

267

268

269

270

271

272

273

274

275

276 as the arithmetic task in which skills-difficulty balance implies the emergence of flow state) compared with low and high difficulty conditions. In addition, they found higher RT in the secondary task during the balanced-difficulty condition relative to the high and low difficulty conditions (Huskey, Craighead, et al., 2018). Interestingly, these behavioural results were accompanied by increased activity in structures associated with cognitive control like the dorsolateral prefrontal cortex (DLPFC), the visual orienting and alertness attentional networks (i.e., superior parietal lobe and dorsal anterior insula, respectively); as well as structures of the reward system (i.e., putamen) in the balanced condition compared to the low and high difficulty conditions, as recorded with fMRI. Subsequent analyses of this database (Huskey, Wilcox, et al., 2018), revealed no greater brain connectivity between cognitive control and reward brain networks during the flow condition compared with the easy and difficult conditions. Further, they explored whether the skills-difficulty balanced condition (i.e., flow condition) was associated with an energetically-efficient topology (as characterized by connections between nodes within a network). In line with their prediction, the flow condition was associated with a lower brain efficiency score (see Rubinov and Sporns, 2010, for a comprehensive explanation of this measure) than the other difficulty conditions, which is interpreted as an index of lower energetic cost.

Since flow state implies focus and full immersion into a primary task, evaluating the performance of a secondary simultaneous task, which should be poor when flow emerges, seems a reasonable way to assess to which extent an individual is experiencing flow. In that sense, the dual-tasking approach may provide an alternative way to explore the behavioural and neural correlates of flow. However, the experiment conducted by Huskey and collaborators (Huskey, Craighead, et al., 2018; Huskey, Wilcox, et al., 2018) is based again on the implicit (and likely erroneous) premise that flow state will necessarily arise if task's difficulty and individual's skills are balanced. Moreover, flow state was not directly measured. Although the authors assessed the participants' intrinsic reward sensitivity, it represents only a single, 
necessary but not sufficient, feature of a situation prone to trigger a flow state. Therefore, the dual-tasking experimental paradigm implemented in this study does not allow one to draw conclusions on whether those increases in RT and the associated changes in brain activity were due to the actual presence of flow state or to mere attentional dynamics while performing tasks varying in difficulty.

\section{Video games studies}

Video games have been frequently used in experimental research to address flow state (Michailidis et al., 2018). This approach is based on the premise that immersive enjoyable video games would be associated with higher chances of triggering flow experiences compared, for example, with performing sums. However, the combination of video games and fMRI has been implemented only two times. In a pioneering study, Klasen and collaborators (2012) asked 13 regular video game players to play a first-person shooter video game while recording their brain activity with fMRI. Researchers analysed the content of the game and classified (according to their subjective criteria) which situations were more related to some of the dimensions of flow (e.g., skills-difficulty balance, focus, clear goals, and control over the situation) in order to identify the moments with higher probability of experiencing flow. During those flow-like situations, increased activity in sensorimotor brain networks (i.e., left primary and secondary somatosensory cortex and motor areas) and the cerebellum was observed. A latter fMRI study (Ju \& Wallraven, 2019) addressed flow state by using a similar video game approach in 31 healthy young participants, finding increased activity in areas involved in the default mode network. Nevertheless, the experimental design of these studies is undermined by the same methodological constraints of the previous reports (e.g., the absence of flow measures, manipulating flow by modifying difficulty level). It should be noted, for example, that investigating the situations with an enhanced likelihood of flow is not equivalent to measuring the flow directly. Likewise, finding activity changes in brain areas related to cognitive processes does not necessarily mean they are linked to flow experience, since an individual can be totally focused on a task and perform well without experiencing flow state. Thus, the brain patterns found in these studies cannot be directly attributed to flow state.

In sum, even if some of the fMRI studies report changes in brain areas and neural systems that might be, in theory, related to flow state, several methodological issues raise doubts about the validity of these findings (i.e., whether the observed brain correlates might be actually attributable to the experience of flow). 


\subsection{Research on oxygen concentration: fNIRS}

311

312

313

314

315

316

317

318

319

320

321

322

323

324

325

326

327

328

329

330

331

332

333

334

335

336

337

338

339

fNIRS is another non-invasive neuroimaging technique to measure cerebral hemodynamic with a high temporal resolution. Note that, although this technique seems particularly pertinent in flow research since it is especially useful to measure prefrontal cortex activity, which has been repeatedly pointed as a key system in the experience of flow (e.g., Dietrich, 2004; Weber et al., 2009), fNIRS does not allow to assess the activity of the striatal reward networks, which is another important component of flow state according to the Synchronization Theory of Flow (Weber et al., 2009). We identified 5 studies addressing the neural correlates of flow by means of fNIRS (de Sampaio Barros et al., 2018; Harmat et al., 2015; Hirao, 2014; Yoshida et al., 2014; Yu et al., 2022).

Three of these studies (de Sampaio Barros et al., 2018; Harmat et al., 2015; Yoshida et al., 2014) implemented the same experimental paradigm, instructing a set of participants to play the classic Tetris video game in different difficulty conditions (modulated by the speed of the game). By relying on the skills-difficulty balance, they inferred that a difficulty-adjusted condition would trigger a flow state. Indeed, in all of these three studies, the self-reported experience of flow state was higher for the difficulty-adjusted condition compared with the low-difficulty condition; however, their results were mixed. On one hand, Yoshida et al. (2014) and de Samapio Barros et al. (2018) reported increased activity in the prefrontal cortex during the adjusted-difficulty (i.e., flow) condition compared with the low-difficulty (i.e., boredom) condition in 15 and 20 young participants, respectively. On the other hand, Harmat and collaborators (2015) found no association between prefrontal cortical oxygenation and flow using the same video game in a sample size of 35 university students.

Similarly, Yu et al. (2022) recently manipulated the level of difficulty of a music game to induce flow states in regular video game players and people with no video game experience, while measuring prefrontal lobe activity with fNIRS. According to their findings, flow ratings correlated linearly with the increase in oxygenated hemoglobin concentration level in prefrontal areas (DLPFC and frontal pole area) in regular and non-regular players. The results from these four studies contrast with the findings reported by Hirao (2014) using a verbal fluency task. In that study, flow experience of 60 young adults was positively correlated with prefrontal hemodynamic suppression. 
This set of studies is particularly relevant for the theoretical debate on the neural mechanisms underlying flow state because they directly tested the THH proposed by Dietrich (2004). In fact, three of the five studies reviewed here showed increased activity in prefrontal regions, which would be contrary to what is proposed by the THH. Nevertheless, it is important to note that these findings are undermined by the same issues of previous studies using fMRI, plus potential biases associated primarily with the measurement of brain activity or the selection of reported results (see Risk of bias, in Supplementary material). Thus, we cannot rule out the THH based on these findings.

\subsection{Research on neural oscillations: EEG}

We identified 11 studies addressing the neural correlates of flow with EEG (4 were case studies) while trying to induce flow state through different experimental protocols (Farrugia et al., 2021; Katahira et al., 2018; Knierim et al., 2018, 2021; Leroy \& Cheron, 2020; Moreno et al., 2020; Núñez Castellar et al., 2019; Shehata et al., 2021; Wolf et al., 2015; Wollseiffen et al., 2016; Yun et al., 2017).

The study of Katahira and collaborators (2018) is particularly relevant as it has attracted the most attention (according to the number of citations) from the scientific community interested in flow state. They employed the arithmetic task developed by Ulrich et al. (2014) in order to induce flow state in 16 participants. After performing the arithmetic task in the three difficulty levels (i.e., low, adjusted and high), participants self-reported their experience of flow. As expected, the subjective ratings of flow state were higher for the adjusted-difficulty condition (i.e., the so-called flow state condition) compared with the easier (i.e., boredom) and difficult (i.e., overload) conditions. Notably, the authors found higher theta power in frontal electrodes during flow and overload conditions, relative to the boredom condition. The authors suggest that the higher activity of theta might be related to the high cognitive control demands required by the task in the flow and overload condition. On the other hand, they found increased alpha power during the flow condition compared with the boredom condition (i.e., low difficulty), that they attributed to the difficulty level of the task, since they also found higher alpha power in the overload condition (i.e, high difficulty) relative to the flow condition (i.e., moderate difficulty). In a similar vein, Knierim and collaborators (2018) 
by using the same arithmetic task $(n=7)$ pointed to alpha and theta brain rhythms at frontocentral locations as potential brain correlates associated with flow state. Very recently, these same authors (Knierim et al., 2021) evaluated the usability of an portable EEG system - BrainComputer-Interface (OpenBCI) platform with c-shaped EEG electrode array (cEEGrid) while 6 participants performed the same mathematical task, and found that, again, more intense subjective flow experiences were associated with moderate levels of alpha activation in temporal areas. Thus, different studies using arithmetic tasks agree in pointing to alpha as the power band associated with flow state. The authors suggest that alpha activity could be involved in a relatively moderate working memory load (Katahira et al., 2018), in sustained attention processes (Knierim et al., 2018), or in a reduction of neural activity due to an efficient use of analytical-verbal reasoning during flow state (Knierim et al., 2021).

\section{Dual-task studies}

Dual-task paradigms have also been used to explore the neural correlates of flow using EEG. As aforementioned, this paradigm is based on the hypothesis that the more attention devoted to the primary task, the less attention will be available for the secondary task, which should result in longer RTs. In line with this hypothesis, Núñez-Castellar and collaborators (2019) asked 18 participants to perform a classic auditory oddball task while playing a video game in three difficulty conditions (i.e., low, adjusted and high), after which the subjects had to answer two questionnaires on flow experience. The authors assumed, as in most of the research on this topic, that skills-difficulty balance would lead participants to a flow state. The results revealed a lower amplitude of the P300 (i.e., an event-related positive deviation of voltage traditionally linked to attentional and executive processes; Polich and Kok, 1995) elicited by the oddball stimulus in central electrodes during the difficulty-adjusted condition (i.e, flow condition) compared with the low (i.e., boredom) and high (i.e., overload) difficult conditions. The authors suggest that the lower amplitude of P300 during flow condition may indicate that subjects were paying less attention to the secondary task, which may be an indirect marker of flow state. In addition, they found increased alpha power in frontal electrodes during the flow condition compared with boredom and overload conditions, which also correlated with longer RTs in the secondary task.

A similar dual-task paradigm was used by Yun and collaborators (2017) to address the EEG correlates of flow state. In this study, 29 healthy young participants were instructed to play a first-person shooting game (primary task) while a sequence of auditory tones was 
randomly presented across time (secondary task). They were asked to ignore these gameunrelated tones while playing during 60 minutes divided into two blocks of 30 minutes varying in difficulty (low and high). Then, participants retrospectively rated their performance and identified periods of flow state and non-flow state (the average duration of flow was $8.31 \pm$ $3.61 \mathrm{~min} ; 13.9 \%$ of total time). The results revealed a lower increase of beta band power evoked by the tones (secondary task) in the anterior cingulate cortex during flow state compared with non-flow state. In addition, this suppressed beta power correlated positively with the selfreported experience of flow. Despite this study is still a preprint, it represents a well-focused approach to study flow state in a laboratory since they used an intrinsically motivating task (first-person video game) to facilitate flow emergence while testing the neural response to a secondary (irrelevant) task, with (relatively) long periods of time without interruptions, a high density EEG system (128 channels) and a relatively high sample size (i.e., 29 participants) with previous experience with video games (average $13.6 \pm 10.1$ hours/week).

\section{Exercise-related studies}

Sport or physical activity has also been used to induce flow state while collecting EEG activity. The first exercise-based approach to the EEG correlates of flow was conducted by Wolf and collaborators (2015). They recruited a set of amateur $(n=15)$ and expert table tennis players $(n=14)$, who performed a task of observation and mental imagery of motor actions. Participants were instructed to observe 40 videos of table tennis serves, imagining that the score was 10-10 (next point would win the match) and that they had to react to that serve. The flow state was measured after the task using a short questionnaire (Flow Kurz Scala; Rheinberg et al., 2003). The authors found that, in the early instances of movement execution, elite players showed higher asymmetry between hemispheres in temporal lobe activity (i.e., higher activity in the temporal lobe of the right hemisphere compared to the temporal lobe of the left hemisphere) relative to the amateurs, which was associated with a greater self-reported experience of flow. They suggest that this pattern of neural activity may indicate a suppression of analytical-verbal activity and irrelevant cognitive processes during the flow state, as well as greater psychomotor efficiency in elite athletes compared to amateurs.

In our view, Wolf and collaborators' (2015) conclusions cannot be drawn from this experiment for several reasons. Firstly, half of the expert participants reported low levels of flow experience (i.e., 7 participants scored below 5 in the 7-point self-rating flow scale) which may cast doubt on whether subjects were actually experiencing flow. Secondly, the many 
differences between elite players and amateurs when faced with a table tennis task even in imagination (e.g., previous experience with similar situations, highly trained motor reactions, opponent movement prediction/anticipation, fitness, etc.) do not allow attributing the observed neural changes to an experience of flow or simply to any of the other between group differences.

Another exercise-related approach was led by Wollseiffen and collaborators (2016), using a dual-task paradigm that combined a primary exercise task (ultramarathon) and a secondary task (arithmetic problem solving). A group of 11 experienced ultramarathoners performed six consecutive 1-hour periods of run, between which EEG (portable device), mood, and cognitive performance measures were collected. These measures were also obtained before and after the 6-hour run. Their results revealed that self-reported flow levels increased after one hour of running and decreased between the first and third hour, remaining relatively stable for the rest of the race. This transient dynamic of flow was not followed by the activity of beta brain rhythm, which decreased after the first hour and remained stable throughout the rest of the race. The authors attribute this phenomenon to a floor effect, suggesting that the activity of the prefrontal cortex during exercise reached its lowest level after the first hour. Notice that there was no correlation between the individual experience of flow and the decrease in frontal beta activity. In addition, they found an increase in global alpha activity after the first 4 hours. Notably, performance in the secondary task did not change over time.

In our opinion, the exercise-based studies described above might be considered (together with the video game studies) among the best approaches to study flow state to date. Physical activity, in particular long-lasting activities such as an ultramarathon, are especially suitable to trigger experiences of flow since they are intrinsically rewarding activities prone to automaticity, performed by highly motivated experts (in those studies), during prolonged periods of time, with clear goals and immediate feedback about performance. However, there are many methodological caveats that diminish the extent to which their findings can be explained by a state of flow or, in contrast, by the physical activity itself (e.g., reliability of low-density portable EEG system, poor signal-to-noise ratio, absence of a proper control condition).

Case studies 
Another way of trying to investigate the oscillatory brain dynamics of flow state is through case studies, in which researchers usually work with highly experienced individuals in a particular task (i.e., individuals who like the task, know very well their skills and can experience a high sense of control of the situation and task performance), which would facilitate the emergence of flow state. Furthermore, case studies provide an opportunity to collect (neural) data in ecological contexts and a fine-grained characterization of the flow experience. We identified 3 relevant case studies using this approach (Farrugia et al., 2021; Leroy \& Cheron, 2020; Moreno et al., 2020). In general, their results point to brain rhythms, especially alpha, beta and gamma, as potential markers of flow state arising in tasks requiring highly specialization such as playing a musical instrument (Farrugia et al., 2021), writing a scientific manuscript (Moreno et al., 2020) or crossing a 15-metre-high tightrope (Leroy \& Cheron, 2020). Interestingly, these studies suggest the use of peripheral psychophysiological measures (e.g., electrocardiogram or galvanic skin conductance) as complementary measures that may help identify and characterize flow state (see Peifer and Tan, 2021, for a review). However, the case studies included in this review are exposed to a moderate risk of bias, which further limits the drawing of conclusions (see Risk of bias, in Supplementary material).

\section{Team flow}

Although most flow research has focused on individual participants, in recent years, there has been a growing interest in the so-called team flow phenomenon (i.e., group flow, collective flow), due to its potential applications in business and sports teams or artistic groups (Pels et al., 2018). Team flow is conceptualized by van den Hout and collaborators (2018) as a state of flow shared by a small group that results from a dynamic and optimal interaction between people while executing interdependent individual tasks. Very recently, Shehata and collaborators (2021) attempted to identify the EEG correlates of this collective flow experience. In their study, 10 pairs of participants matched on the basis of their game skills and musical tastes played a music rhythm game. Flow level was manipulated by scrambling the music of the game (i.e., a reversed and shuffled version of the music was played, modifying the intrinsic enjoyment dimension of flow, instead of the skill-difficulty balance) and by whether participants could see or not their partner. As a result, participants simulated playing an instrument in 3 different experimental conditions (i.e., team flow, flow only and team only) while their brain activity was recorded with EEG. Interestingly, they validated their experimental manipulation with subjective ratings of the flow state and objective measures of 
task-irrelevant auditory-evoked potentials —in line with the dual-task paradigms hypothesis above, e.g., Núñez-Castellar et al. (2019)—. The results of Shehata and collaborators (2021) pointed to higher beta and gamma power in the middle temporal cortex (MTC) and enhanced neural synchrony as correlates of team flow. Furthermore, they found lower beta and gamma power in the PFC in the flow conditions compared with the no-flow conditions, which is consistent with previous observations with fMRI (Ulrich et al, 2016a, 2016b, 2014).

Taken together, the evidence from EEG studies suggest that flow state might be linked to changes in specific brain rhythms (i.e., theta, alpha, beta and gamma). However, the experimental designs and paradigms implemented in these studies do not allow to elucidate whether these changes in brain activity can be considered a consequence of experiencing flow or a mere neural correlate of a closely related state (e.g., full attention), which does not necessarily imply to be in flow.

\subsection{Research on brain stimulation: tDCS}

Throughout the literature on the neural basis of the flow state only two studies have used tDCS to facilitate the emergence of flow state and explore its neural correlates (Gold \& Ciorciari, 2019; Ulrich et al., 2018). Both studies represent an innovative approach, in an attempt to establish causal rather than correlational relationships. These studies tried to induce flow state in healthy participants by increasing or decreasing the excitability of regions of the cerebral cortex that would be involved in flow, according to the scarce existing literature (e.g., Ulrich et al., 2016a, 2016b, 2014). In a first study, Ulrich and collaborators (2018) recruited 22 participants to perform an arithmetic task with three difficulty conditions (i.e., low, adjusted, high) while applying tDCS in combination with perfusion MRI. Participants performed the task in three separate experimental sessions under anodal, cathodal or sham stimulation at Fpz, an electrode location associated with the MPFC. Contrary to what they expected, the subjective experience of flow was similar across the different tDCS conditions. However, in a further exploratory analysis, participants were divided into two subgroups (i.e., high-flow and lowflow) according to the median split of the flow index obtained in the sham condition. When brain activity of both groups was compared, they observed that the low-flow group showed higher deactivation of the right amygdala associated with an increase in the flow index compared to the high-flow group. The authors concluded that individuals less susceptible to 
528

529

530

531

532

533

534

535

536

537

538

539

540

541

542

543

544

545

546

547

548

549

550

551

552

553

554

555

556

557

558

flow state may benefit more than high-flow individuals from active tDCS in the MPFC. However, due to the post-hoc exploratory nature of these analyses, and the fact that Ulrich and collaborators (2018) found no differences in the reported flow state between the sham, anodal and cathodal stimulation conditions, no conclusions about the role of the MPFC can be drawn from these results.

Gold and Ciorciari (2019) investigated whether increased excitability in the right parietal cortex and decreased excitability in the left DLPFC (anodal tDCS at P6 and cathodal at F3) would result in greater flow experience, as measured by the Flow State Scale (Jackson \& Marsh, 1996). To do so, they conducted two experiments: one with expert video game players $(n=11)$, and another one with non-experts $(n=21)$. Both groups played Tetris at three difficulty levels (i.e., low, adaptive, and high). Researchers found that both groups (regular gamers and non-gamers) reported higher scores of flow experience after the tDCS stimulation compared with the sham condition. According to previous research, anodal parietal stimulation appears to increase connectivity within neural networks, including inferior and superior parietal along with the cerebellum, which has been linked to learning outcomes (Hunter et al., 2015). On the other hand, DLPFC inhibition seems to enhance dynamic balance between explicit and implicit systems (Eichenbaum \& Cohen, 2004). In this line, the authors suggest that increased right parietal activity together with decreased activity in the left DLPFC may foster flow experience.

To sum up, while tDCS studies could help clarify some of the contradictions found in the literature on the neural basis of flow state, the paucity of research to date (only two studies so far) does not allow drawing any definitive conclusion about the involvement of specific areas in flow state. In addition, it is important to highlight that tDCS has courted significant controversy in the last years due to failed replication attempts, unknown physiological basis, and variability in outcomes, resulting in skepticism regarding its reported effects (e.g., Filmer et al., 2020; Parkin et al., 2019).

\section{Discussion}

The purpose of the present study was to systematically review the existent empirical evidence on the neural correlates of flow state. We found a total of 25 studies using a wide range of strategies to experimentally address flow state and its neural dynamics and revised 
them from a critical perspective, an approach that had not been pursued before in the literature on the potential neural mechanisms of flow. After scrutinizing the experimental paradigms implemented and their resulting findings, it is concluded that the extant evidence is sparse and inconclusive, with major methodological shortcomings that prevent us from drawing solid conclusions about the neural correlates of flow state. Nevertheless, these investigations were pioneering in the study of flow state from a neuroscientific perspective and their findings help speculate about the potential neural correlates of flow that future studies may confirm.

In general, the studies using neuroimaging techniques while (trying to) inducing flow seem to converge on the key role of structures linked to attention, executive function and reward systems, giving to the anterior brain areas (e.g., the DLPC) a crucial role in the experience of flow. However, the dynamics of these brain regions during flow state are inconsistent across studies. Studies using fMRI report mixed patterns of activation and deactivation of specific frontal areas such as the IFG and the medial prefrontal cortex (Ulrich et al., 2014, 2016a, 2018), the DLPC (Huskey, Craighead, et al., 2018; Huskey, Wilcox, et al., 2018) or the default mode network (Ju \& Wallraven, 2019). In a similar vein, findings from fNIRS studies are especially inconsistent, showing increased (de Sampaio Barros et al., 2018; Yoshida et al., 2014) and decreased prefrontal activity (Hirao, 2014) during flow state, as well as no significant prefrontal activity changes (Harmat et al., 2015). Regarding brain oscillatory activity, EEG studies point to increased power of particular brain rhythms such as theta (Katahira et al., 2018; Knierim et al., 2018) and alpha (Knierim et al., 2018; Núñez Castellar et al., 2019) at frontal locations during flow state. Finally, tDCS studies also suggest a central role of the prefrontal cortex in flow state although their results are mixed (Gold \& Ciorciari, 2019; Ulrich et al., 2018).

Notably, the absence of consistent overlaps between the brain regions activated during (presumably) flow states in the few studies included in the present systematic review is not surprising. On the one hand, extremely different methodological approaches have been used to induce flow, especially with regard to experimental tasks, including: arithmetic tasks, video games, a verbal fluency test, writing a $\mathrm{PhD}$ thesis, a mental imagery task, running a marathon or playing an instrument, among others. On the other hand, flow is a complex state which is made up of different subcomponents - as indicated in the Introduction of this review, according to the author of the original Theory of Flow, there are 9 different subcomponents of flow state (Csikszentmihalyi, 1975)—. As mentioned above (see Results), some approaches 
591

592

593

594

595

596

597

598

599

600

601

602

603

604

605

606

607

608

609

610

611

612

613

614

615

616

617

618

619

620

621

622

have focused on manipulating only one of these subcomponents of flow (e.g., skill-difficulty balance, intrinsic reward). Therefore, studies that have induced different aspects of the flow state can be expected to lead to different outcomes. These two major weaknesses in the existing literature on the neural basis of flow make it particularly difficult to cluster studies, compare their results, and above all, to draw firm conclusions.

As noted in the Introduction of this article, the two theoretical models (i.e., THH and STF) that would account for the results summarized above stand for radically different positions on the neural dynamics associated with the state of flow. On one hand, the THH argues for a suppression of frontal activity during flow state which would reduce interference of the explicit processing (e.g., self-referential thought) and facilitate implicit processing (i.e., automatized processes). Several studies reviewed here seem to provide partial support for this theory (Gold \& Ciorciari, 2019; Hirao, 2014; Katahira et al., 2018; Núñez Castellar et al., 2019; Ulrich et al., 2014, 2016a, 2016b, 2018; Wollseiffen et al., 2016; Yun et al., 2017). However, even assuming these studies were actually capturing the neural signatures of flow state, their findings would suggest specific brain patterns associated with flow rather than a general deactivation of frontal areas. On the other hand, the STF, based on findings from neuroimaging studies on flow-like activities (e.g., hypnosis and meditation) showing strong brain frontal activity (e.g., Newberg and Iversen, 2003), advocates for increased neural synchronization between neural attention networks (i.e., executive, alerting and orienting) during flow state. This principle of energetically-efficient brain functioning is a tentative (and highly speculative) account to explain why flow is perceived as neither physically nor mentally depleting and effortless despite the fact that the tasks commonly used to induce flow require a moderate-tohigh level of difficulty (Huskey, Craighead, et al., 2018; Huskey, Wilcox, et al., 2018). While the STF seems to be supported by the results reported by several studies reviewed here (de Sampaio Barros et al., 2018; Huskey, Craighead, et al., 2018; Huskey, Wilcox, et al., 2018; Katahira et al., 2018; Klasen et al., 2012; Wolf et al., 2015; Yun et al., 2017), the methodological limitations of these studies together with findings from some accounts that seem to contradict this model (e.g., Huskey et al., 2018b, 2018a) cast doubts on it.

The Internal Model of Flow proposed by Gold and Ciorciari (2021) attempts to reconcile these two seemingly contrary theories to explain most of the mixed evidence to date, relying on the role of cerebellar regions. This model would be supported, for instance, by the findings reported in Ulrich et al. (2016a), who observed activity in the cerebellum, premotor 
623

624

625

626

627

628

629

630

631

632

633

634

635

636

637

638

639

640

641

642

643

644

645

646

647

648

649

650

651

652

regions and pre-supplementary motor cortex during flow state. Also compatible with the model are the observations made by Klasen et al. (2012), who found activity in the cerebellum and some regions of the reward system (e.g., putamen).

In any case, as already pointed out, despite the fact these studies are paving the road to unveil the brain mechanisms supporting the flow experience, the evidence to date is sparse, unreliable and inconclusive to allow any proper theoretical debate.

\section{The triad of (erroneous) inferences when studying flow}

Whatever the theoretical interpretation of the results reviewed here, one should have in mind that a main part of this literature relies on three crucial inferences that, in our opinion, limit the validity of their findings.

Inference 1: Flow state arises in situations where the task's difficulty matches the skills of the individual. It is well-known that skills-difficulty balance is a necessary condition for flow state to arise, but not sufficient since it represents only one feature of the experience of flow. For the flow state to emerge, a task must also be regarded by the individual as critical and challenging (but attainable), with immediate feedback about performance, carried out in a well-known taskrelated scenario and during prolonged periods of time without interruption (Nakamura \& Csikszentmihalyi, 2014). Further, even if all these facilitating conditions are met it is conceivable that flow state will not arise (i.e., we often face tasks whose difficulty matches our skills and we do not experience flow). Thus, it seems unreasonable to assume that flow state may arise performing a non-critical unmotivating task (i.e., sums) in an unrelated scenario (e.g., fMRI scanner) with continuous interruptions.

Inference 2: The observed changes in brain activity are due to flow state. Even assuming these difficulty-based paradigms actually lead to flow state, it cannot be concluded that the changes in brain activity observed during the flow condition are due to an experience of flow rather than to a difference in the level of task's difficulty across conditions. These paradigms often compare brain activity patterns collected during a moderate difficulty condition adjusted to the subject's skill level (i.e., flow condition), with a low (i.e., boredom condition) or a high difficulty condition (i.e., overload condition). The observed changes (e.g., changes in the activity of prefrontal areas; e.g., Hirao, 2014; Katahira et al., 2018; Ulrich et al., 2016a; 
653 Wollseiffen et al., 2016) may therefore be simply due to a variation in the difficulty level, as noted by Ulrich and collaborators (2014). In fact, task difficulty manipulation induces an effortbased cost-benefit analysis which is computed by prefrontal areas (Egner, 2009; Rushworth et al., 2004). Moreover, in a recent study, Mallat and collaborators (2020), who used the task difficulty manipulation approach, found that the highest level of effort mobilisation occurred at an intermediate level of task difficulty, compared to very low or very high difficulty levels (i.e., effortful control is maximal for moderate levels of difficulty). Consequently, any variation in prefrontal activity found in the balanced skill-difficulty condition compared to low and high difficulty conditions can be attributed to effort variations and not to a flow state. The absence of an appropriate control condition prevents attributing the observed brain dynamics to the state of flow (see Future directions section for recommendations on this issue).

Inference 3: If the brain area $X$ is active, then the cognitive process $Y$ is engaged. The reversal inference is one of the most prominent inferential strategies in cognitive neuroscience (Nathan \& Del Pinal, 2017; Poldrack, 2006), and the study of the neural basis of flow has not been exempted from it. In this particular case, this inference arises when the observed changes in brain regions or systems previously associated with flow-related cognitive processes are directly considered as a sign of the presence of flow state. Given the multi-functionality of most brain areas and neural systems, it seems unreasonable to assume that all the observed brain activity changes found during flow state would be selective of it, rather than a concomitant neural correlated of any other related process. A clear example of that inference in flow research is the key role of the cerebellum put forward by the Internal Model of Flow (Gold \& Ciorciari, 2021). While some studies found increased activity in the cerebellum during flow state (Klasen et al., 2012; Ulrich et al., 2016a), such direct association would be questioned when considering recent accounts of the cerebellum's involvement in cognitive and emotional processes, in the same way that it regulates sensorimotor and vestibular control (e.g., Schmahmann et al., 2019).

In sum, when delving into what flow state is and how its correlates (i.e., neural, cognitive, and phenomenological) can be measured, one may ultimately consider whether we are indeed ready to identify and quantify reliable neural correlates of the flow state. In the next section, potential solutions to these three major flaws in flow state research are directly addressed. 
686

687

688

689

690

691

692

693

694

695

696

697

698

699

700

701

702

703

704

705

706

707

708

709

710

711

712

713

714

715

As mentioned along these lines, due to the intrinsic characteristics of the flow state, one might argue that it is unlikely that flow could be triggered in controlled laboratory conditions where neural activity can be reliably recorded. However, several considerations regarding experimental design and flow measurements may provide useful avenues for future studies in flow research.

First, a paradigm shift is needed, from the traditional experimental paradigm for inducing flow state through arithmetic tasks to more intrinsically motivating tasks such as video games or physical exercise. Video games are particularly suitable for studying the flow state in a laboratory because the scenario can be quite similar to a real situation, with low motor activity, high levels of enjoyment and immersion. Moreover, the movement associated with physical exercise and the need to be carried in ecological settings makes it more complicated to collect quality data on electrical activity (e.g., portable EEG systems can be used, but not high-density EEG or other types of neuroimaging techniques), a constraint that is not present when video games are used as an experimental task. In addition, video games are often played over extended periods of time, facilitating and increasing the likelihood of flow.

Second, there is a need for studies with large samples of experts since they are more likely to experience prolonged states of flow, as well as studies in which individuals are trained in particular activities to experience flow state more easily. Notably, larger sample sizes in general are necessary - more than half of the studies included in the present review have samples of less than 20 participants - , so that studies attempting to observe the neural correlates of flow have sufficient statistical power to detect the effect, if present. In this sense, the number of video game players is growing every year and would facilitate the collection of large sample sizes of experts, which again points to video games as a promising activity to experimentally address flow state under controlled conditions.

Third, implementing repeated measures designs, with several experimental sessions in different days with the same subjects, would not only increase the chances of each subject to experiencing flow, but would also enhance the individual's familiarity with the situation, presumably fostering the emergence of flow. Moreover, it would provide a more appropriate control condition for flow experiments. That is, by having brain recordings of each individual during different sessions (or periods during the same session) with similar task conditions (e.g., 
in terms of difficulty), one may compare brain activity patterns in periods when the individual experienced the flow state with those when they did not.

Fourth, the use of methodologies that allow ongoing tracking of the subjective experience of flow state without continuous interruptions, so that the transient fluctuations of the subject's state throughout the task can be captured. Recently, a novel phenomenological method has been developed for capturing continuous subjective experiences: Temporal experience tracing (Jachs, 2021). This method requires participants to retrospectively graph the intensity of an experience along a particular phenomenological dimension (e.g., flow, attention, boredom) over time. This would reduce the number of interruptions during the task (asking for self-reporting of several flow-related items), facilitating the occurrence and maintenance of flow state. Furthermore, it would allow a fine-grained characterization of flow state temporal fluctuations.

Fifth, the use of portable systems to measure brain activity (e.g., wearable Dry-EEG headset) may help collect neural data in ecological situations where it is easier to trigger an experience of flow. In fact, in one of the most recent studies included in the review, Knierim and collaborators (Knierim et al., 2021) employed an interesting portable EEG device, a 3D printed Brain-Computer-Interface platform with c-shaped EEG electrode array, which, if validated with a larger experimental sample, could be a promising tool for research on flow states in facilitating contexts.

Sixth, something intriguing we found in the literature of flow state is the unjustified misrepresentation of women. From the 25 studies included in this systematic review, only 14 included female participants (72.6\% of the total sample were men). Some of them even justify the selective inclusion of men to reduce putative sex differences due to hormonal alterations during the menstrual cycle (Ulrich et al., 2014, 2018). However, the hormone-brain-cognition relationship is not as straightforward as one might think (Fine, 2010, 2017; Weigard et al., 2021), and brain differences between males and females appear trivial and population-specific based on recent accounts (Eliot et al., 2021; Rippon, 2019). In any case, to be representative of the whole population, studies should include male and female participants. We therefore encourage researchers to design gender-balanced studies in the future to reduce this gap in flow literature. 
And last, but not least, future studies on the neural correlates of the flow state should

747

748

749

750 avoid falling into the erroneous inferences that have weakened the existing literature so far. The use of tasks, procedures and contexts that facilitate the emergence of the flow state, such as those stated above, must be accompanied by direct subjective (e.g., questionnaires) and objective, albeit indirect (e.g., auditory evoked potentials), measures of the flow state. Moreover, these measures should be implemented together with sophisticated control measures of other variables that may be influencing changes in brain activity - e.g., effortful control (see Inference 2 above), which could be controlled by measuring pre-ejection period (PEP) with electrocardiogram (EKG), a reliable and valid index of mental effort mobilization (Mallat et al., 2020)—. This will allow stronger links to be established between the recorded brain activity and the flow state. Furthermore, the use of reverse inference (i.e., proposing which cognitive processes may be involved in the flow state based solely on observed activity in specific areas) should be avoided.

\subsection{Limitations}

The present systematic review is undermined by the small number of studies addressing this topic. Only 25 studies were included, which were based on a wide range of paradigms and analytical approaches. This small number of studies, together with the high heterogeneity across them and the moderate risk of bias estimated for many of them (see Risk of bias, in Supplementary material), drastically ruled out the possibilities of using meta-analytic techniques since its potential results would be meaningless. Moreover, most of these studies present important methodological limitations that considerably hamper the scope of our conclusions.

The main objectives and methods (i.e., search protocol) were not pre-registered before the systematic review was carried out, a practice that would have contributed to greater transparency and avoidance of bias (Stewart et al., 2012), as well as the avoidance of possible unintentional duplication of effort to collect neural correlates of the flow state. The reason why this review was not pre-registered is that it was initially carried out as the first author's (CA) final undergraduate project, under the supervision of LC and DS. Given the quality of that first manuscript and the relevance of the topic, the authors decided to submit it to a peer-review 
journal, after updating the list of papers with a second search (performed both by CA and LC) and further rounds of amendments.

\subsection{Final conclusion}

The present systematic review synthesises and critically assesses the extant scientific evidence on the neural correlates of flow state, questioning for the first time in the literature the validity and reliability of the findings reported in the experiments attempting to capture the brain signatures of flow experience. In general, studies addressing this topic are scarce, showing large heterogeneity in the methods and inconsistency in the outcomes, which limits any theoretical debate or potential application. Despite the absence of conclusive evidence, it is important to note that these studies pave the way for future work and help speculate about the potential neural signatures of flow state to drive future research on this topic.

\section{Acknowledgments}

This manuscript is dedicated to the memory of Professor Mihály Csíkszentmihályi (1934-2021), whose pioneering work on flow state inspired countless ideas during the course of this project and will undoubtedly continue inspiring future generations of flow researchers.

\section{Funding}

This research was supported by a predoctoral fellowship from the Spanish Ministry of Education and Vocational Training to CA (20CO1/012863), a research project grant from the Spanish Ministry of Science and Innovation to DS (PID2019-105635GB-I00), a postdoctoral fellowship from the Regional Government of Andalusia to LFC (DOC_00225). We thank Chiara Avancini for her thoughtful comments and insightful suggestions on the manuscript. 


\section{References}

Arsalidou, M., \& Taylor, M. J. (2011). Is $2+2=4$ ? Meta-analyses of brain areas needed for numbers and calculations. Neuroimage, 54(3), 2382-2393. https://doi.org/10.1016/j.neuroimage.2010.10.009

Baldo, J. V., \& Dronkers, N. F. (2007). Neural correlates of arithmetic and language comprehension: A common substrate? Neuropsychologia, 45(2), 229-235. https://doi.org/10.1016/j.neuropsychologia.2006.07.014

Butkovic, A., Ullén, F., \& Mosing, M. A. (2015). Personality related traits as predictors of music practice: Underlying environmental and genetic influences. Personality and Individual Differences, 74, 133-138. https://doi.org/10.1016/j.paid.2014.10.006

Cranston, S., \& Keller, S. (2013). Increasing the meaning quotient of work. McKinsey Quarterly, 1(48-59).

Csikszentmihalhi, M. (2020). Finding flow: The psychology of engagement with everyday life. Hachette UK.

Csikszentmihalyi, M. (1975). Beyond Boredom and Anxiety: The Notion of Flow in Work and Play. San Francisco: Jossey Press.

Csikszentmihalyi, M., Abuhamdeh, S., \& Nakamura, J. (2021). Flow. Natur \& Kultur Allmänlitteratur.

de Manzano, Ö., Cervenka, S., Jucaite, A., Hellenäs, O., Farde, L., \& Ullén, F. (2013). Individual differences in the proneness to have flow experiences are linked to dopamine D2-receptor availability in the dorsal striatum. NeuroImage, 67, 1-6. https://doi.org/10.1016/j.neuroimage.2012.10.072

de Sampaio Barros, M. F., Araújo-Moreira, F. M., Trevelin, L. C., \& Radel, R. (2018). Flow experience and the mobilization of attentional resources. Cognitive, Affective, \& Behavioral Neuroscience, 18(4), 810-823. https://doi.org/10.3758/s13415-018-06064

Dietrich, A. (2004). Neurocognitive mechanisms underlying the experience of flow. Consciousness and Cognition, 13(4), 746-761. https://doi.org/10.1016/j.concog.2004.07.002

Egner, T. (2009). Prefrontal cortex and cognitive control: Motivating functional hierarchies. Nature Neuroscience, 12(7), 821-822. https://doi.org/10.1038/nn0709-821 https://doi.org/10.1038/nn0709-821

Eichenbaum, H., \& Cohen, N. J. (2004). From conditioning to conscious recollection: Memory systems of the brain. Oxford University Press on Demand. 
Eliot, L., Ahmed, A., Khan, H., \& Patel, J. (2021). Dump the "dimorphism": Comprehensive synthesis of human brain studies reveals few male-female differences beyond size. Neuroscience \& Biobehavioral Reviews. https://doi.org/10.1016/j.neubiorev.2021.02.026

Farrugia, N., Lamouroux, A., Rocher, C., Bouvet, J., \& Lioi, G. (2021). FBeta and Theta Oscillations Correlate With Subjective Time During Musical Improvisation in Ecological and Controlled Settings: A Single Subject Study. Frontiers in Neuroscience. 15:626723. https://doi.org/10.3389/fnins.2021.626723

Filmer, H. L., Mattingley, J. B., \& Dux, P. E. (2020). Modulating brain activity and behaviour with tDCS: Rumours of its death have been greatly exaggerated. Cortex, 123, 141-151. https://doi.org/10.1016/j.cortex.2019.10.006

Fine, C. (2010). Delusions of gender: How our minds, society, and neurosexism create difference. WW Norton \& Company.

Fine, C. (2017). Testosterone rex: Unmaking the myths of our gendered minds. Icon Books.

Flett, M. R. (2015). Is Flow Related to Positive Feelings or Optimal Performance? Path Analysis of Challenge-Skill Balance and Feelings. Sport Science Review, 24.

Gold, J., \& Ciorciari, J. (2019). A Transcranial Stimulation Intervention to Support Flow State Induction. Frontiers in Human Neuroscience, 13, 274. https://doi.org/10.3389/fnhum.2019.00274

Gold, J., \& Ciorciari, J. (2021). A neurocognitive model of flow states and the role of cerebellar internal models. Behavioural Brain Research, 113244. https://doi.org/10.1016/j.bbr.2021.113244

Gruber, O., Indefrey, P., Steinmetz, H., \& Kleinschmidt, A. (2001). Dissociating neural correlates of cognitive components in mental calculation. Cerebral Cortex, 11(4), 350-359. https://doi.org/10.1093/cercor/11.4.350

Gyurkovics, M., Kotyuk, E., Katonai, E. R., Horvath, E. Z., Vereczkei, A., \& Szekely, A. (2016). Individual differences in flow proneness are linked to a dopamine D2 receptor gene variant. Consciousness and Cognition, 42, 1-8. https://doi.org/10.1016/j.concog.2016.02.014

Harmat, L., de Manzano, Ö., Theorell, T., Högman, L., Fischer, H., \& Ullén, F. (2015). Physiological correlates of the flow experience during computer game playing. International Journal of Psychophysiology, 97(1), 1-7. https://doi.org/10.1016/j.ijpsycho.2015.05.001

Heo, J., Lee, Y., Pedersen, P. M., \& McCormick, B. P. (2010). Flow Experience in the Daily Lives of Older Adults: An Analysis of the Interaction between Flow, Individual 

Aging / La Revue Canadienne Du Vieillissement, 29(3), 411-423. https://doi.org/10.1017/S0714980810000395

Hirao, K. (2014). Prefrontal hemodynamic responses and the degree of flow experience among occupational therapy students during their performance of a cognitive task. Journal of Educational Evaluation for Health Professions, 11. https://doi.org/10.3352/jeehp.2014.11.24

Hunter, M. A., Coffman, B. A., Gasparovic, C., Calhoun, V. D., Trumbo, M. C., \& Clark, V. P. (2015). Baseline effects of transcranial direct current stimulation on glutamatergic neurotransmission and large-scale network connectivity. Brain Research, 1594, 92107. https://doi.org/10.1016/j.brainres.2014.09.066

Huskey, R., Craighead, B., Miller, M. B., \& Weber, R. (2018). Does intrinsic reward motivate cognitive control? A naturalistic-fMRI study based on the synchronization theory of flow. Cognitive, Affective, \& Behavioral Neuroscience, 18(5), 902-924. https://doi.org/10.3758/s13415-018-0612-6

Huskey, R., Wilcox, S., \& Weber, R. (2018). Network Neuroscience Reveals Distinct Neuromarkers of Flow During Media Use. Journal of Communication, 68(5), 872895. https://doi.org/10.1093/joc/jqy043

Jachs, B. (2021). The neurophenomenology of meditative states: Introducing temporal experience tracing to capture subjective experience states and their neural correlates. University of Cambridge.

Jackson, S. A., \& Eklund, R. C. (2002). Assessing flow in physical activity: The flow state scale-2 and dispositional flow scale-2. Journal of Sport and Exercise Psychology, 24(2), 133-150.

Jackson, S. A., \& Marsh, H. W. (1996). Development and validation of a scale to measure optimal experience: The Flow State Scale. Journal of Sport and Exercise Psychology, 18(1), 17-35. https://doi.org/10.1123/jsep.18.1.17

Ju, U., \& Wallraven, C. (2019). Manipulating and decoding subjective gaming experience during active gameplay: A multivariate, whole-brain analysis. NeuroImage, 188, 113. https://doi.org/10.1016/j.neuroimage.2018.11.061

Katahira, K., Yamazaki, Y., Yamaoka, C., Ozaki, H., Nakagawa, S., \& Nagata, N. (2018). EEG Correlates of the Flow State: A Combination of Increased Frontal Theta and Moderate Frontocentral Alpha Rhythm in the Mental Arithmetic Task. Frontiers in Psychology, 9, 300. https://doi.org/10.3389/fpsyg.2018.00300 
Khoshnoud, S., Alvarez Igarzábal, F., \& Wittmann, M. (2020). Peripheral-physiological and neural correlates of the flow experience while playing video games: A comprehensive review. PeerJ, 8, e10520. https://doi.org/10.7717/peerj.10520

Klasen, M., Weber, R., Kircher, T. T., Mathiak, K. A., \& Mathiak, K. (2012). Neural contributions to flow experience during video game playing. Social Cognitive and Affective Neuroscience, 7(4), 485-495. https://doi.org/10.1093/scan/nsr021

Knierim, M. T., Berger, C., \& Reali, P. (2021). Open-source concealed EEG data collection for Brain-computer-interfaces-neural observation through OpenBCI amplifiers with around-the-ear cEEGrid electrodes. Brain-Computer Interfaces, 8(4), 161-179. https://doi.org/10.48550/arXiv.2102.00414

Knierim, M. T., Nadj, M., Hariharan, A., \& Weinhardt, C. (2018). Flow Neurophysiology in Knowledge Work: Electroencephalographic Observations from Two Cognitive Tasks: Proceedings of the 5th International Conference on Physiological Computing Systems, 42-53. https://doi.org/10.5220/0006926700420053

Kong, J., Wang, C., Kwong, K., Vangel, M., Chua, E., \& Gollub, R. (2005). The neural substrate of arithmetic operations and procedure complexity. Cognitive Brain Research, 22(3), 397-405. https://doi.org/10.1016/j.cogbrainres.2004.09.011

Lambert, J., \& Csikszentmihalyi, M. (2020). Facilitating or foiling flow: The role of momentary perceptions of feedback. The Journal of Positive Psychology, 15(2), 208219. https://doi.org/10.1080/17439760.2019.1578893

Leroy, A., \& Cheron, G. (2020). EEG dynamics and neural generators of psychological flow during one tightrope performance. Scientific Reports, 10(1), 12449. https://doi.org/10.1038/s41598-020-69448-3

Lewis, G. (2013). Emotion and disaffection with school mathematics. Research in Mathematics Education, 15(1), 70-86. https://doi.org/10.1080/14794802.2012.756636

Lewis, P. A., Critchley, H. D., Rotshtein, P., \& Dolan, R. J. (2007). Neural correlates of processing valence and arousal in affective words. Cerebral Cortex, 17(3), 742-748. https://doi.org/10.1093/cercor/bhk024

MacDonald, R., Byrne, C., \& Carlton, L. (2006). Creativity and flow in musical composition: An empirical investigation. Psychology of Music, 34(3), 292-306. https://doi.org/10.1177/0305735606064838

Makris, N., Oscar-Berman, M., Jaffin, S. K., Hodge, S. M., Kennedy, D. N., Caviness, V. S., Marinkovic, K., Breiter, H. C., Gasic, G. P., \& Harris, G. J. (2008). Decreased Volume of the Brain Reward System in Alcoholism. Biological Psychiatry, 64(3), 192-202. https://doi.org/10.1016/j.biopsych.2008.01.018 
Mallat, C., Cegarra, J., Calmettes, C., \& Capa, R. L. (2020). A Curvilinear Effect of Mental Workload on Mental Effort and Behavioral Adaptability: An Approach With the PreEjection Period. Human Factors, 62(6), 928-939. https://doi.org/10.1177/0018720819855919

McGaugh, J. L. (2004). The amygdala modulates the consolidation of memories of emotionally arousing experiences. Annu. Rev. Neurosci., 27, 1-28. https://doi.org/10.1146/annurev.neuro.27.070203.144157

Michailidis, L., Balaguer-Ballester, E., \& He, X. (2018). Flow and immersion in video games: The aftermath of a conceptual challenge. Frontiers in Psychology, 9, 1682. https://doi.org/10.3389/fpsyg.2018.01682

Moher, D., Shamseer, L., Clarke, M., Ghersi, D., Liberati, A., Petticrew, M., Shekelle, P., \& Stewart, L. A. (2015). Preferred reporting items for systematic review and metaanalysis protocols (PRISMA-P) 2015 statement. Systematic Reviews, 4(1), 1-9. https://doi.org/10.1186/2046-4053-4-1

Moreno, M., Schnabel, R., Lancia, G., \& Woodruff, E. (2020). Between text and platforms: A case study on the real-time emotions \& psychophysiological indicators of video gaming and academic engagement. Education and Information Technologies, 25(3), 2073-2099. https://doi.org/10.1007/s10639-019-10031-3

Mosing, M. A., Pedersen, N. L., Cesarini, D., Johannesson, M., Magnusson, P. K. E., Nakamura, J., Madison, G., \& Ullén, F. (2012). Genetic and Environmental Influences on the Relationship between Flow Proneness, Locus of Control and Behavioral Inhibition. PLOS ONE, 7(11), e47958. https://doi.org/10.1371/journal.pone.0047958

Nakamura, J., \& Csikszentmihalyi, M. (2009). Flow theory and research. Handbook of Positive Psychology, 195-206.

Nakamura, J., \& Csikszentmihalyi, M. (2014). The concept of flow. In Flow and the foundations of positive psychology (pp. 239-263). Springer.

Nathan, M. J., \& Del Pinal, G. (2017). The future of cognitive neuroscience? Reverse inference in focus. Philosophy Compass, 12(7), e12427. https://doi.org/10.1111/phc3.12427

Newberg, A. B., \& Iversen, J. (2003). The neural basis of the complex mental task of meditation: Neurotransmitter and neurochemical considerations. Medical Hypotheses, 61(2), 282-291. https://doi.org/10.1016/S0306-9877(03)00175-0

Núñez Castellar, E. P., Antons, J., Marinazzo, D., \& Van Looy, J. (2019). Mapping attention during gameplay: Assessment of behavioral and ERP markers in an auditory oddball task. Psychophysiology, 56(7), e13347. https://doi.org/10.1111/psyp.13347 
Ottiger, B., Van Wegen, E., Keller, K., Nef, T., Nyffeler, T., Kwakkel, G., \& Vanbellingen, T. (2021). Getting into a "Flow" state: A systematic review of flow experience in neurological diseases. Journal of Neuroengineering and Rehabilitation, 18(1), 1-21. https://doi.org/10.1186/s12984-021-00864-w

Parkin, B. L., Bhandari, M., Glen, J. C., \& Walsh, V. (2019). The physiological effects of transcranial electrical stimulation do not apply to parameters commonly used in studies of cognitive neuromodulation. Neuropsychologia, 128, 332-339. https://doi.org/10.1016/j.neuropsychologia.2018.03.030

Peifer, C., \& Tan, J. (2021). The Psychophysiology of Flow Experience. In Advances in Flow Research (pp. 191-230). Springer. https://doi.org/10.1007/978-3-030-53468-4_8

Pels, F., Kleinert, J., \& Mennigen, F. (2018). Group flow: A scoping review of definitions, theoretical approaches, measures and findings. PloS One, 13(12), e0210117. https://doi.org/10.1371/journal.pone.0210117

Poldrack, R. A. (2006). Can cognitive processes be inferred from neuroimaging data? Trends in Cognitive Sciences, 10(2), 59-63. https://doi.org/10.1016/j.tics.2005.12.004

Polich, J., \& Kok, A. (1995). Cognitive and biological determinants of P300: An integrative review. Biological Psychology, 41(2), 103-146. https://doi.org/10.1016/03010511(95)05130-9

Posner, M. I., Inhoff, A. W., Friedrich, F. J., \& Cohen, A. (1987). Isolating attentional systems: A cognitive-anatomical analysis. Psychobiology, 15(2), 107-121. https://doi.org/10.3758/BF03333099

Posner, M. I., \& Rothbart, M. K. (2007). Research on Attention Networks as a Model for the Integration of Psychological Science. Annual Review of Psychology, 58(1), 1-23. https://doi.org/10.1146/annurev.psych.58.110405.085516

Rheinberg, F., Vollmeyer, R., \& Engeser, S. (2003). Die erfassung des flow-erlebens.

Rippon, G. (2019). The Gendered Brain: The new neuroscience that shatters the myth of the female brain. Random House.

Ross, S. R., \& Keiser, H. N. (2014). Autotelic personality through a five-factor lens: Individual differences in flow-propensity. Personality and Individual Differences, 59, 3-8. https://doi.org/10.1016/j.paid.2013.09.029

Rubinov, M., \& Sporns, O. (2010). Complex network measures of brain connectivity: Uses and interpretations. Neuroimage, 52(3), 1059-1069. https://doi.org/10.1016/j.neuroimage.2009.10.003 
Rushworth, M. F. S., Walton, M. E., Kennerley, S. W., \& Bannerman, D. M. (2004). Action sets and decisions in the medial frontal cortex. Trends in Cognitive Sciences, 8(9), 410-417. https://doi.org/10.1016/j.tics.2004.07.009

Schmahmann, J. D., Guell, X., Stoodley, C. J., \& Halko, M. A. (2019). The theory and neuroscience of cerebellar cognition. Annual Review of Neuroscience, 42, 337-364. https://doi.org/10.1146/annurev-neuro-070918-050258

Schmidt, J. A., Shernoff, D. J., \& Csikszentmihalyi, M. (2014). Individual and Situational Factors Related to the Experience of Flow in Adolescence. In M. Csikszentmihalyi (Ed.), Applications of Flow in Human Development and Education: The Collected Works of Mihaly Csikszentmihalyi (pp. 379-405). Springer Netherlands. https://doi.org/10.1007/978-94-017-9094-9_20

Shehata, M., Cheng, M., Leung, A., Tsuchiya, N., Wu, D.-A., Tseng, C., Nakauchi, S., \& Shimojo, S. (2021). Team Flow Is a Unique Brain State Associated with Enhanced Information Integration and Interbrain Synchrony. ENeuro, 8(5). https://doi.org/10.1523/ENEURO.0133-21.2021

Smallwood, J., Bernhardt, B. C., Leech, R., Bzdok, D., Jefferies, E., \& Margulies, D. S. (2021). The default mode network in cognition: A topographical perspective. Nature Reviews Neuroscience, 22(8), 503-513. https://doi.org/10.1038/s41583-021-00474-4

Stavrou, N. A., Jackson, S. A., Zervas, Y., \& Karteroliotis, K. (2007). Flow experience and athletes' performance with reference to the orthogonal model of flow. The Sport Psychologist, 21(4), 438-457. https://doi.org/10.1123/tsp.21.4.438

Sterne JAC, Savović J, Page MJ, Elbers RG, Blencowe NS, Boutron I, Cates CJ, Cheng H-Y, Corbett MS, Eldridge SM, Hernán MA, Hopewell S, Hróbjartsson A, Junqueira DR, Jüni P, Kirkham JJ, Lasserson T, Li T, McAleenan A, Reeves BC, Shepperd S, Shrier I, Stewart LA, Tilling K, White IR, Whiting PF, Higgins JPT. RoB 2: a revised tool for assessing risk of bias in randomised trials. BMJ 2019; 366: 14898.

Stewart, L., Moher, D., \& Shekelle, P. (2012). Why prospective registration of systematic reviews makes sense. Systematic Reviews, 1(1), 1-4. https://doi.org/10.1186/20464053-1-7

Swann, C., Keegan, R. J., Piggott, D., \& Crust, L. (2012). A systematic review of the experience, occurrence, and controllability of flow states in elite sport. Psychology of Sport and Exercise, 13(6), 807-819. https://doi.org/10.1016/j.psychsport.2012.05.006

Ullén, F., Harmat, L., Theorell, T., \& Madison, G. (2016). Flow and Individual Differences A Phenotypic Analysis of Data from More than 10,000 Twin Individuals. In L. Harmat, F. Ørsted Andersen, F. Ullén, J. Wright, \& G. Sadlo (Eds.), Flow Experience: Empirical Research and Applications (pp. 267-288). Springer International Publishing. https://doi.org/10.1007/978-3-319-28634-1_17 
Ulrich, M., Keller, J., \& Grön, G. (2016a). Neural signatures of experimentally induced flow experiences identified in a typical fMRI block design with BOLD imaging. Social Cognitive and Affective Neuroscience, 11(3), 496-507. https://doi.org/10.1093/scan/nsv133

Ulrich, M., Keller, J., \& Grön, G. (2016b). Dorsal Raphe Nucleus Down-Regulates Medial Prefrontal Cortex during Experience of Flow. Frontiers in Behavioral Neuroscience, 10. https://doi.org/10.3389/fnbeh.2016.00169

Ulrich, M., Keller, J., Hoenig, K., Waller, C., \& Grön, G. (2014). Neural correlates of experimentally induced flow experiences. Neuroimage, 86, 194-202. https://doi.org/10.1016/j.neuroimage.2013.08.019

Ulrich, M., Niemann, J., Boland, M., Kammer, T., Niemann, F., \& Grön, G. (2018). The neural correlates of flow experience explored with transcranial direct current stimulation. Experimental Brain Research, 236(12), 3223-3237. https://doi.org/10.1007/s00221-018-5378-0

van den Hout, J. J. J., Davis, O. C., \& Weggeman, M. C. D. P. (2018). The Conceptualization of Team Flow. The Journal of Psychology, 152(6), 388-423. https://doi.org/10.1080/00223980.2018.1449729

van der Linden, D., Tops, M., \& Bakker, A. B. (2021). Go with the flow: A neuroscientific view on being fully engaged. European Journal of Neuroscience, 53(4), 947-963. https://doi.org/10.1111/ejn.15014

Weber, R., Tamborini, R., Westcott-Baker, A., \& Kantor, B. (2009). Theorizing flow and media enjoyment as cognitive synchronization of attentional and reward networks. Communication Theory, 19(4), 397-422. https://doi.org/10.1111/j.14682885.2009.01352.x

Weigard, A., Loviska, A. M., \& Beltz, A. M. (2021). Little evidence for sex or ovarian hormone influences on affective variability. Scientific Reports, 11(1), 1-12. https://doi.org/10.1038/s41598-021-00143-7

Wolf, S., Brölz, E., Keune, P. M., Wesa, B., Hautzinger, M., Birbaumer, N., \& Strehl, U. (2015). Motor skill failure or flow-experience? Functional brain asymmetry and brain connectivity in elite and amateur table tennis players. Biological Psychology, 105, 95105. https://doi.org/10.1016/j.biopsycho.2015.01.007

Wollseiffen, P., Schneider, S., Martin, L. A., Kerhervé, H. A., Klein, T., \& Solomon, C. (2016). The effect of $6 \mathrm{~h}$ of running on brain activity, mood, and cognitive performance. Experimental Brain Research, 234(7), 1829-1836. https://doi.org/10.1007/s00221-016-4587-7 
1096

1097

1098

1099

1100

1101

1102

1103

1104

1105

1106

1107

1108

1109

1110

Yoshida, K., Sawamura, D., Inagaki, Y., Ogawa, K., Ikoma, K., \& Sakai, S. (2014). Brain activity during the flow experience: A functional near-infrared spectroscopy study. Neuroscience Letters, 573, 30-34. https://doi.org/10.1016/j.neulet.2014.05.011

Yu, D., Wang, S., Song, F., Liu, Y., Zhang, S., Wang, Y., Xie, X., \& Zhang, Z. (2022). Research on user experience of the video game difficulty based on flow theory and fNIRS. Behaviour \& Information Technology, 0(0), 1-17. https://doi.org/10.1080/0144929X.2022.2043442

Yun, K., Doh, S., Carrus, E., Wu, D.-A., \& Shimojo, S. (2017). Neural correlates of flow using auditory evoked potential suppression. ArXiv Preprint ArXiv:1711.06967.

Zago, L., Petit, L., Turbelin, M.-R., Andersson, F., Vigneau, M., \& Tzourio-Mazoyer, N. (2008). How verbal and spatial manipulation networks contribute to calculation: An fMRI study. Neuropsychologia, 46(9), 2403-2414. https://doi.org/10.1016/j.neuropsychologia.2008.03.001

列

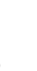

.

.


1112 


\section{Table 1}

Characteristic of the studies investigating neural correlates of flow state

\begin{tabular}{|c|c|c|c|c|c|c|}
\hline Study & $\mathbf{N}(\mathbf{f})$ & $\begin{array}{l}\text { Age } \\
\text { (SD/range) }\end{array}$ & Sample & Task & Flow measures & Main findings \\
\hline \multicolumn{7}{|l|}{ fMRI } \\
\hline Ulrich et al., 2014 & 27 & $23 \pm 2.3$ & University students & Arithmetic task & $\begin{array}{l}\text { Flow Index ( } 9 \text { Likert- } \\
\text { scale items) }\end{array}$ & $\begin{array}{l}\text { Increased activity in the left IFG, putamen and posterior cortical } \\
\text { regions, and decreased activity in the MPFC, the left AMY, } \\
\text { hippocampus and parahippocampal gyrus in the flow condition. }\end{array}$ \\
\hline $\begin{array}{l}\text { Ulrich, Keller \& } \\
\text { Grön, 2016a }\end{array}$ & 23 & $24 \pm 2.7$ & University students & Arithmetic task & $\begin{array}{l}\text { SFPQ } \\
\text { Flow Index }\end{array}$ & $\begin{array}{l}\text { Increased activity in the IFG, left putamen and posterior cortical } \\
\text { regions, and decreased activity in the MPFC, PCC and AMY in the } \\
\text { flow condition. }\end{array}$ \\
\hline $\begin{array}{l}\text { Ulrich, Keller \& } \\
\text { Grön, 2016b }\end{array}$ & 23 & $24 \pm 2.7$ & University students & Arithmetic task & $\begin{array}{l}\text { SFPQ } \\
\text { Flow Index }\end{array}$ & $\begin{array}{l}\text { Stronger down-regulatory influence of the DRN on the MPFC when } \\
\text { participants experienced flow. }\end{array}$ \\
\hline $\begin{array}{l}\text { Huskey, Craighead, } \\
\text { Miller \& Weber, } \\
2018\end{array}$ & $18(14)$ & 22.8 & University students & $\begin{array}{l}\text { P: video game } \\
\text { S: visual } R T \text { task }\end{array}$ & $\begin{array}{l}\text { Autotelic personality } \\
\text { subscale of the AES }\end{array}$ & $\begin{array}{l}\text { Increased activity in DLPFC, superior parietal lobe, precentral gyrus, } \\
\text { dorsal anterior insula and putamen in the flow condition. }\end{array}$ \\
\hline $\begin{array}{l}\text { Huskey, Wilcox \& } \\
\text { Weber, } 2018\end{array}$ & $18(14)$ & 22.8 & University students & $\begin{array}{l}\text { P: video game } \\
\text { S: visual RT task }\end{array}$ & $\begin{array}{l}\text { Autotelic personality } \\
\text { subscale of the AES }\end{array}$ & $\begin{array}{l}\text { Lower energetic cost in the flow condition. No greater connectivity } \\
\text { between cognitive control and reward networks in the flow condition. }\end{array}$ \\
\hline Klasen et al., 2012 & 13 & 23 & Regular video gamers & Video game & - & $\begin{array}{l}\text { Increased activity in sensorimotor networks and the cerebellum during } \\
\text { game moments with higher probability of flow state. }\end{array}$ \\
\hline Ju \& Wallraven, 2019 & 31 & $24.8 \pm 3.6$ & Regular video gamers & Video game & GEQ & $\begin{array}{l}\text { Correlation between flow and the activity of regions related to visual } \\
\text { and spatial processing and attentional processes. Negative correlation } \\
\text { with the activity of the DMN. }\end{array}$ \\
\hline \multicolumn{7}{|l|}{ fNIRS } \\
\hline Yoshida et al., 2014 & $15(9)$ & $22.0 \pm 1$ & University students & Video game & $\begin{array}{l}\text { Flow state scale for } \\
\text { occupational tasks }\end{array}$ & Increased activity in VLPFC, FPA, and DLPFC during flow condition. \\
\hline Harmat et al., 2015 & $35^{\mathrm{a}}$ & $27.8 \pm 5.4$ & University students & Video game & FSS-2 & No association between frontal cortical oxygenation and flow scores. \\
\hline Hirao, 2014 & $60(22)$ & $19.5 \pm 0.9$ & University students & Verbal fluency test & Flow Questionnaire & $\begin{array}{l}\text { Negative correlation between the average oxygenated hemoglobin in } \\
\text { the prefrontal cortex and flow scores. }\end{array}$ \\
\hline
\end{tabular}




\begin{tabular}{|c|c|c|c|c|c|c|}
\hline $\begin{array}{l}\text { de Sampaio Barros, } \\
2018\end{array}$ & $20(7)$ & $26.4 \pm 4.8$ & Adult volunteers & Video game & Flow Short Scale & $\begin{array}{l}\text { Flow condition correlated with higher concentration of oxygenated } \\
\text { hemoglobin in regions of the frontoparietal network. }\end{array}$ \\
\hline Yu et al., 2022 & $40(18)$ & $19-26$ & University students & Video game & Flow Short Scale & $\begin{array}{l}\text { Flow level correlated linearly with the increase in oxygenated } \\
\text { hemoglobin concentration level in DLPFC and FPA. }\end{array}$ \\
\hline \multicolumn{7}{|l|}{ EEG } \\
\hline Katahira et al., 2018 & $16(6)$ & $21.9 \pm 1.1$ & University students & Arithmetic task & Flow index & $\begin{array}{l}\text { Correlation between flow and theta activity in frontal electrodes, and } \\
\text { alpha activity in fronto-central electrodes. }\end{array}$ \\
\hline Knierim et al., 2018 & 7 & $21-30$ & University students & $\begin{array}{l}\text { Writing } \mathrm{PhD} \text { thesis } \\
\text { Arithmetic task }\end{array}$ & Flow Short Scale & $\begin{array}{l}\text { Increased theta and alpha activity in frontal electrodes as task difficulty } \\
\text { increases. }\end{array}$ \\
\hline Knierim et al., 2021 & 6 & 24-30 & University students & Arithmetic task & Flow Short Scale & Increased alpha temporal activity in the flow condition. \\
\hline $\begin{array}{l}\text { Nuñez-Castellar et } \\
\text { al., } 2019\end{array}$ & $18(9)$ & $28.5 \pm 4.6$ & Casual video gamers & $\begin{array}{l}\text { P: video game } \\
\text { S: auditory oddball }\end{array}$ & $\begin{array}{l}\text { Flow Short Scale } \\
\text { Flow Questionnaire }\end{array}$ & $\begin{array}{l}\text { Lower amplitude of P } 300 \text { in the flow condition and increased frontal } \\
\text { alpha in the flow condition. }\end{array}$ \\
\hline Yun et al., 2017 & $29(5)$ & $23.5 \pm 3.4$ & Regular video gamers & $\begin{array}{l}\text { P: video game } \\
\text { S: random sounds }\end{array}$ & $\begin{array}{l}\text { Reported flow level } \\
\text { (Think Aloud design) }\end{array}$ & $\begin{array}{l}\text { Lower increase of beta band power evoked by the random sounds in the } \\
\text { ACC during flow compared to non-flow state. }\end{array}$ \\
\hline Wolf et al., 2015 & $29(9)$ & 23.3 & $\begin{array}{l}\text { Amateur and expert } \\
\text { table tennis players }\end{array}$ & $\begin{array}{l}\text { Mental motor } \\
\text { imagery }\end{array}$ & Flow Short Scale & $\begin{array}{l}\text { Flow correlates with less temporal activity in the left hemisphere and } \\
\text { increased activity in right temporal hemisphere in the expert group. }\end{array}$ \\
\hline $\begin{array}{l}\text { Wollseiffen et al., } \\
2016\end{array}$ & $11(5)$ & $36.5 \pm 7$ & $\begin{array}{l}\text { Ultramarathon } \\
\text { runners }\end{array}$ & $\begin{array}{l}\text { P: 6h-running } \\
\text { S: Arithmetic task }\end{array}$ & $\begin{array}{l}\text { FSS-2 short version } \\
\text { FSS-2 long version }\end{array}$ & $\begin{array}{l}\text { Decreased frontal beta activity and increased flow after the first hour. } \\
\text { No correlation between flow state and beta or alpha after the first hour. }\end{array}$ \\
\hline Farrugia et al., 2021 & 1 & 53 & Musician & Improvisation & $\begin{array}{l}\text { Concentration } \\
\text { STR }\end{array}$ & $\begin{array}{l}\text { Positive correlation between fast perceived passage of time and brain } \\
\text { rhythms (theta and beta) activity. }\end{array}$ \\
\hline Moreno et al., 2019 & $2(1)$ & - & $\begin{array}{l}\text { Regular video gamer } \\
\text { Academic writer }\end{array}$ & $\begin{array}{l}\text { Video game } \\
\text { Scientific article }\end{array}$ & FSS & $\begin{array}{l}\text { Increased beta and gamma activity during flow condition in both } \\
\text { participants. }\end{array}$ \\
\hline $\begin{array}{l}\text { Leroy \& Chéron, } \\
2020\end{array}$ & 1 & - & Tightrope walker & Crossing a tightrope & Flow Short Scale & Increased alpha activity during the flow period. \\
\hline Shehata et al., 2021 & $15(10)$ & $18-35$ & - & Video game & $\begin{array}{l}\text { Flow Index (6 Likert- } \\
\text { scale items) }\end{array}$ & Higher beta and gamma power in MTC during team flow condition. \\
\hline
\end{tabular}




\begin{tabular}{|c|c|c|c|c|c|c|}
\hline Ulrich et al., 2018 & 22 & $24.9 \pm 2.2$ & University students & Arithmetic task & $\begin{array}{l}\text { Flow Index } \\
\text { SFPQ }\end{array}$ & No effects of TDCS in the MPFC in subjective experience of flow. \\
\hline $\begin{array}{l}\text { Gold \& Ciorciari, } \\
2019\end{array}$ & $\begin{array}{l}\text { E1: } 11 \\
\text { E2: } 21 \\
(11)\end{array}$ & $\begin{array}{l}\mathrm{E} 1: 2 \pm 7.1 \\
\mathrm{E} 2: 30.9\end{array}$ & $\begin{array}{l}\text { E1: regular video } \\
\text { gamers } \\
\text { E2: non-gamers }\end{array}$ & Video games & FSS & $\begin{array}{l}\text { Decreased DLPFC activity and increased right parietal cortex activity } \\
\text { associated with flow state in regular video gamers and non-gamers. }\end{array}$ \\
\hline
\end{tabular}

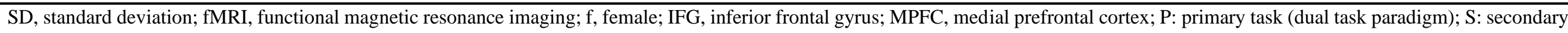

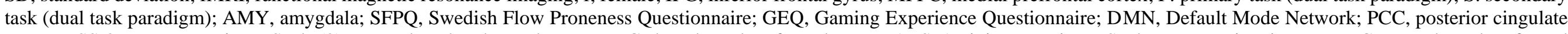

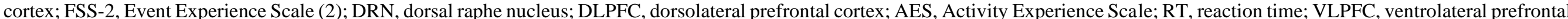

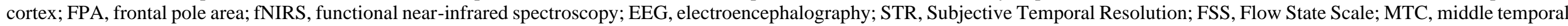
cortex; TDCS, transcranial direct current stimulation; E1, experiment number 1; E2, experiment number 2.

${ }^{a}$ Sex is not specified 


\title{
$\underline{\text { Supplementary material }}$
}

\section{The brain in flow: a systematic review on the neural basis of the flow state}

\author{
Clara Alameda $^{1,2}$, Daniel Sanabria ${ }^{1,2} \&$ Luis F. Ciria ${ }^{1,2}$ \\ ${ }^{1}$ Mind, Brain \& Behavior Research Center, University of Granada, Spain \\ ${ }^{2}$ Department of Experimental Psychology, University of Granada, Spain
}

\section{EXCLUDED STUDIES}

\section{Excluded studies for not meeting the inclusion criteria $(k=24)$ :}

No peer review:

Bishop, A., MacNeil, E., \& Izzetoglu, K. (2021, July). Cognitive Workload Quantified by Physiological Sensors in Realistic Immersive Settings. In International Conference on Human-Computer Interaction (pp. 119-133). Springer, Cham.

Bombeke, K., Dongen, A. V., Durnez, W., Anzolin, A., Almgren, H., All, A., ... \& Núñez Castellar, E. P. (2018, July). Do not disturb: psychophysiological correlates of boredom, flow and frustration during VR gaming. In International Conference on Augmented Cognition (pp. 101-119). Springer, Cham.

Burns, A., \& Tulip, J. (2017, August). Detecting flow in games using facial expressions. In 2017 IEEE Conference on Computational Intelligence and Games (CIG) (pp. 45-52). IEEE.

Carofiglio, V., De Carolis, B. N., \& D'Errico, F. (2019, September). A BCI-based Assessment of a Player's State of Mind for Game Adaptation. In GHITALY@ CHItaly.

Cassani, R., Tiwari, A., Posner, I., Afonso, B., \& Falk, T. H. (2020, October). Initial investigation into neurophysiological correlates of argentine tango flow states: a case study. In 2020 IEEE International Conference on Systems, Man, and Cybernetics (SMC) (pp. 3478-3483).

Gupta, K., Zhang, Y., Pai, Y. S., \& Billinghurst, M. (2021). VR-Wizard: Towards an EmotionAdaptive Experience in VR. In SIGGRAPH Asia 2021 Posters (pp. 1-2).

Knierim, M. T., Nadj, M., \& Weinhardt, C. (2019). Flow and Optimal Difficulty in the Portable EEG: On the Potentiality of using Personalized Frequency Ranges for State Detection. In CHIRA (pp. 183-190).

Labonté-Lemoyne, É., Léger, P. M., Resseguier, B., Bastarache-Roberge, M. C., Fredette, M., Sénécal, S., \& Courtemanche, F. (2016, May). Are we in flow neurophysiological correlates of flow states in a collaborative game. In Proceedings of the $2016 \mathrm{CHI}$ 
Conference Extended Abstracts on Human Factors in Computing Systems (pp. 19801988).

Li, M., Chen, C., Hua, C., \& Guan, X. (2019, May). CFlow: A learning-based compressive flow statistics collection scheme for SDNs. In ICC 2019-2019 IEEE International Conference on Communications (ICC) (pp. 1-6). IEEE.

Mann, S., Defaz, D., Abdulazim, T., Lam, D., Alford, M., Stairs, J., \& Mann, C. (2019, June). Encephalogames TM (brain/mind games): inclusive health and wellbeing for people of all abilities. In 2019 IEEE Games, Entertainment, Media Conference (GEM) (pp. 1-10). IEEE.

Plotnikov, A., Stakheika, N., De Gloria, A., Schatten, C., Bellotti, F., Berta, R., ... \& Ansovini, F. (2012, July). Exploiting real-time EEG analysis for assessing flow in games. In 2012 IEEE 12th International Conference on Advanced Learning Technologies (pp. 688689). IEEE.

Sinha, A., Gavas, R., Chatterjee, D., Das, R., \& Sinharay, A. (2015, October). Dynamic assessment of learners' mental state for an improved learning experience. In 2015 IEEE frontiers in education conference (FIE) (pp. 1-9). IEEE.

Wang, C. C., \& Hsu, M. C. (2013). Flow experience and challenge-skill balance in e-learning. In 2013 Pacific Asia Conference on Information Systems (PACIS).

No specific measures of flow state (e.g., measures of creativity, performance, attention, or costumer experience):

Budnik-Przybylska, D., Kastrau, A., Jasik, P., Kaźmierczak, M., Doliński, Ł., Syty, P., \& Bertollo, M. (2021). Neural oscillation during mental imagery in sport: an olympic sailor case study. Frontiers in Human Neuroscience, 15.

Kwon, S. H., Lee, Y. J., \& Kwon, Y. J. (2020). Why do students fall into webtoon viewing while they give up mathematics? - An fMRI study. International Journal of Knowledge and Learning, 13(3), 201-213.

McMahan, T., Parberry, I., \& Parsons, T. D. (2015). Evaluating player task engagement and arousal using electroencephalography. Procedia Manufacturing, 3, 2303-2310.

Rothlein, D., DeGutis, J., \& Esterman, M. (2018). Attentional fluctuations influence the neural fidelity and connectivity of stimulus representations. Journal of cognitive neuroscience, 30(9), 1209-1228.

Škola, F., Rizvić, S., Cozza, M., Barbieri, L., Bruno, F., Skarlatos, D., \& Liarokapis, F. (2020). Virtual reality with 360-video storytelling in cultural heritage: Study of presence, engagement, and immersion. Sensors, 20(20), 5851.

Wang, C. C., \& Hsu, M. C. (2014). An exploratory study using inexpensive electroencephalography (EEG) to understand flow experience in computer-based instruction. Information \& Management, 51(7), 912-923. 
Weber, R., Alicea, B., Huskey, R., \& Mathiak, K. (2018). Network dynamics of attention during a naturalistic behavioral paradigm. Frontiers in human neuroscience, 12, 182.

No specific measures of brain activity during flow state (i.e., correlations between flow state and brain activity are not analyzed):

Wang, Z., Li, M., \& Yuan, J (2022). An Empirical Study of Geography Learning on Students' Emotions and Motivation in Immersive Virtual Reality. In Frontiers in Education (p. 74). Frontiers.

Wu, S. F., Lu, Y. L., \& Lien, C. J. (2021). Detecting students' flow states and their construct through electroencephalogram: Reflective flow experiences, balance of challenge and skill, and sense of control. Journal of Educational Computing Research, 58(8), 15151540 .

Wu, S. F., Lu, Y. L., \& Lien, C. J. (2021). Measuring effects of technological interactivity levels on flow with electroencephalogram. IEEE Access, 9, 85813-85822.

Yang, X., Lin, L., Cheng, P. Y., Yang, X., \& Ren, Y. (2019). Which EEG feedback works better for creativity performance in immersive virtual reality: The reminder or encouraging feedback?. Computers in Human Behavior, 99, 345-351. 


\section{RISK OF BIAS}

Table summarizing risk of bias assessment of the 25 selected studies with a slightly modified version of the RoB 2 tool (revised Cochrane risk of bias tool for randomised trials):

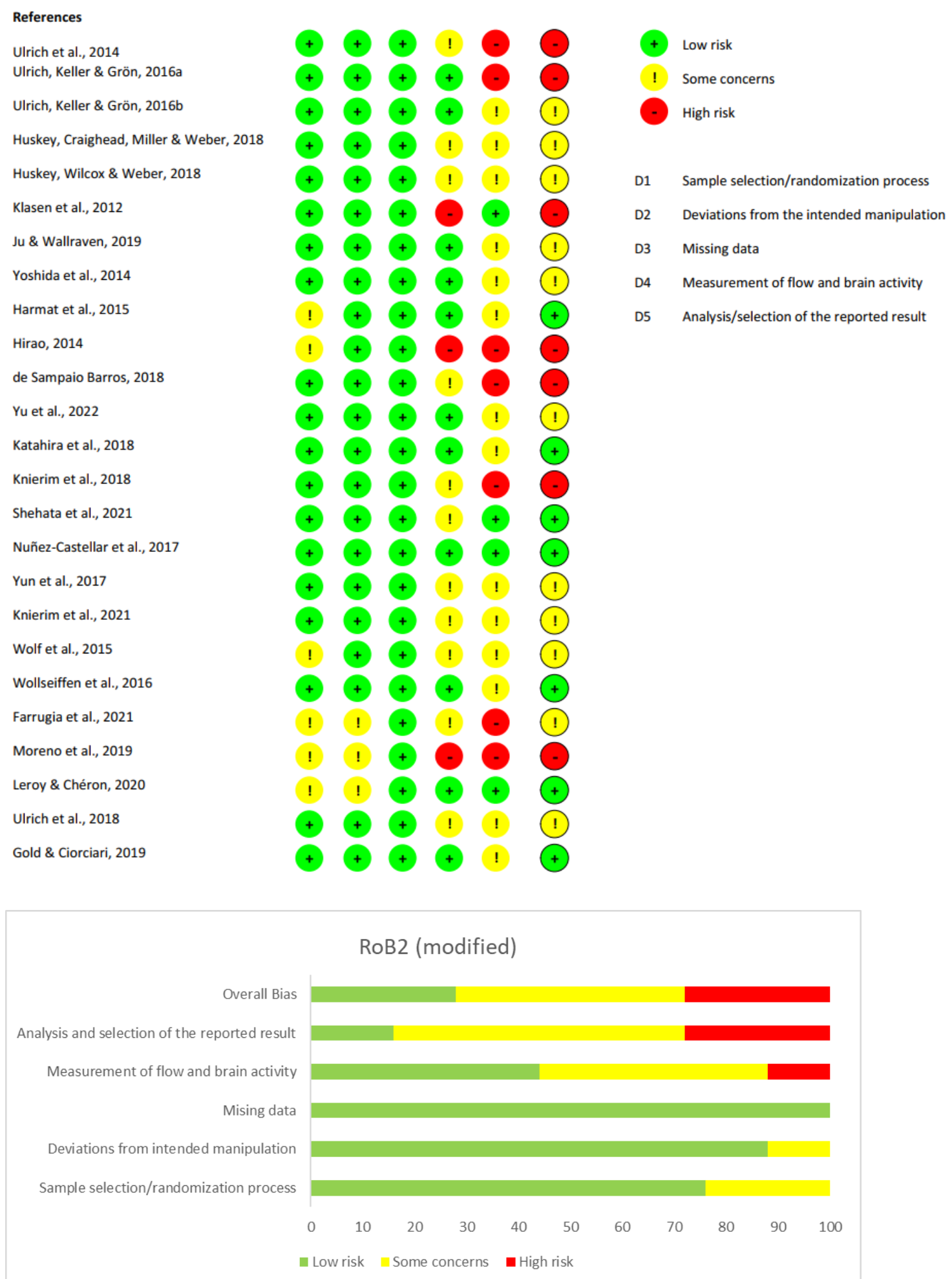




\section{FUNDING SOURCES}

\section{Table summarizing the funding sources of the 25 selected articles:}

All authors in the 25 studies declare that they have no conflict of interest.

\section{Study $\quad$ Funding sources}

\begin{tabular}{|c|c|}
\hline Ulrich et al., 2014 & $\begin{array}{l}\text { German Research Foundation (DFG) to Johannes Keller [grant } \\
\text { number KE 913/5-1] }\end{array}$ \\
\hline Ulrich, Keller \& Grön & $\begin{array}{l}\text { German Research Foundation (DFG) to Johannes Keller [g } \\
\text { number KE 913/5-1] }\end{array}$ \\
\hline
\end{tabular}

Ulrich, Keller \& Grön, 2016b German Research Foundation (DFG) to JK [grant number KE 913/51]

Huskey, Craighead, Miller \& $-^{\mathrm{a}}$ Weber, 2018

Huskey, Wilcox \& Weber, 2018 The University of California Santa Barbara George D. McCune Dissertation Fellowship (to R. H.), University of California Santa Barbara Brain Imaging Center, the University of California Santa Barbara Academic Senate (grant AS-8-588817-19941-7 to R. W.), and the Institute for Social, Behavioral and Economic Research (grant ISBG-SS17WR-8-447631-19941 to R. W.)

Klasen et al., 2012

German Research Foundation (Deutsche Forschungsgemeinschaft (DFG), IRTG 1328; \& MA 2631-4) and the IZKF Aachen (N2-3)

Ju \& Wallraven, 2019

Basic Science Research Program through the National Research Foundation of Korea funded by the Ministry of Science, ICT \& Future planning (NRF-2015S1A5A8018, NRF2017M3C7A1041817) and the Brain Korea 21plus program through the National Research Foundation of Korea (NRF) funded by the Ministry of Education

Yoshida et al., 2014

Harmat et al., 2015

Sven and Dagmar Salén Foundation, the Swedish Scientific Council (521-2010-3195), the Freemasons in Sweden Foundation for Children's Welfare, and the Bank of Sweden Tercentenary Foundation (M11-0451:1)

Hirao, 2014

de Sampaio Barros, 2018

Marcelo Felipe de Sampaio Barros was supported by a scholarship from the 820 Cogn Affect Behav Neurosci (2018) 18:810-823 CAPES Foundation of the Ministry of Education of Brazil, BrasíliaDF 70040-020, Brazil (No. 99999.010663/2014-02). Rémi Radel was supported by a grant of the Agence Nationale de la Recherche (ANR-JCJC, 2013-069) 
Yu et al., 2022

Katahira et al., 2018

Knierim et al., 2018

Knierim et al., 2021

Nuñez-Castellar et al., 2019

Yun et al., 2017

Wolf et al., 2015

Wollseiffen et al., 2016

Farrugia et al., 2021

Moreno et al., 2019

Leroy \& Chéron, 2020

Shehata et al., 2021

Ulrich et al., 2018
Social Science Planning Fund Program of Shandong [grant number: 18CCXJ23]

Japan Society for the Promotion of Science (Grant Number JP15H05347), MEXT-Supported Program for the Strategic Research Foundation at Private Universities, 2014-2018 (Grant Number S1411038) and Center of Innovation Program from Japan Science and Technology Agency, JST

$-\mathrm{a}$

$-$

Postdoctoral grant from the Ghent University special research fund (DPO/AWS-AAP/PP01/KD/30267635) and short-stay mobility grant COST-Quality of Experience in Multimedia Systems and Services (QUALINET)

JST-ERATO, JST-CREST, Tamagawa-Caltech gCOE programs, Grant-in-Aid for Scientific Research (Kakenhi) in Japan, and Basic Science Research Program through the National Research Foundation of Korea (NRF) funded by the Ministry of Education, Science and Technology (2013R1A6A3A03020772)

German research associations: the German research association, DFG, (BI 195/67-1 and Kosellek), the German Center for Diabetes Research (DZD, 01G/0925), the Eva and Horst Köhler Foundation and the VW Foundation.

$-a$

Britanny region, grant SAD-MultiGSP (Award ID: SAD-2019) and the Finistere department, grant MultiGSP

$-a$

The Belgian National Fund for Scientific Research (FNRS), the Research Funds, the Leibu Fund of the Université Libre de Bruxelles and the Université de Mons (Belgium), and the European Commission Project EACEA 34/2018-2019 'Wires Crossed - Head, Heart, Balance / WCHHB'

The Program for Promoting the Enhancement of Research Universities funded to Toyohashi University of Technology and Grants-in-Aid for Scientific Research (Fostering Joint International Research (B), Grant Number 18KK0280) (M.S. and S.N.), Sponsored Research by Qneuro, Inc. (M.S. and S.S.), Translational Research Institute through NASA Cooperative Agreement NNX16AO69A (M.S. and S.S.), and Japan Science and Technology (JST)-CREST Grant JPMJCR14E4 (to S.S.). The University of Hong Kong Postgraduate Scholarship Program. The University of Hong Kong General Research Fund and the Cooperative Research Project Program of the Research Institute of Electrical Communication, Tohoku University. Australian Research Council Discovery Projects Grants DP180104128 and DP18010039, and Australian Government Research Training Program Scholarship 
Gold \& Ciorciari, 2019

This research did not receive any specific grant from funding agencies in the public, commercial, or not-for-profit sectors

${ }^{a}$ Information on funding sources is not available. 
